\title{
EXTENSION PROBLEM FOR QUASI ADDITIVE SET FUNCTIONS AND RADON-NIKODYM DERIVATIVES
}

\author{
BY \\ LAMBERTO CESARI( $\left.{ }^{(}\right)$
}

Introduction. In the preceding paper [1] we have introduced by means of axioms a concept of quasi additive vector-valued set functions $\phi(I)$ $=\left(\phi_{1}, \cdots, \phi_{k}\right)$ in a class $\{I\}$ of sets $I$ of a space $A$, a concept of mesh $\delta(D)$ of certain finite collections $D$ of sets $I$, and we have shown that an integral $\Im(f, T, \phi)$ of a function $f(p, q)$ over a variety $T: p=p(w), w \in A$, with respect to the quasi additive set function $\phi(I)$, can be obtained by a standard process of limit over quasi additive set functions as $\delta(D) \rightarrow 0$. Here $f(p, q), p \in E_{n}$, $q \in E_{k}$, is a function with $f(p, t q)=t f(p, q)$ for all $t \geqq 0$, satisfying usual hypotheses of continuity.

In the present paper, we discuss the problem of extension of quasi additive set functions $\phi, \phi_{r},\|\phi\|,\left|\phi_{r}\right|, \phi_{r}^{+}, \phi_{r}^{-}$into measures $\nu, \nu_{r}, \mu, \mu_{r}, \mu_{r}^{+}, \mu_{r}^{-}$in $A$, and consequent representation theorem for the integral $\Im$.

First the axioms underlying quasi additive set functions and mesh are reworded $(\$ 1)$ in such a way to take into account the sets $G$ of a given class (5) of "open" sets of $A$. Then a first extension of the functions $\phi, \phi_{r},\|\phi\|$, etc., is made into the class $\$$ of open sets $G(\$ 2)$. It is shown by examples that, in the present generality, the extension, say $V$ of $\|\phi\|$, does not satisfy necessarily simple expected properties as $V\left(\sum G_{i}\right) \leqq \sum V\left(G_{i}\right)$ and others. Nevertheless, the addition of further and very natural axioms allows to prove some of these properties as theorems. Finally ( $\$ 3)$, by a further slight reinforcement of the same axioms, we prove that the extensions $\nu, \nu_{r}, \mu, \mu_{r}, \mu_{r}^{+}, \mu_{r}^{-}$in the minimum $\sigma$-ring $\mathfrak{B}$ of sets $B \subset A, \mathfrak{B}$ containing $(\mathcal{B}$, are measures, and that $\nu_{r}=\nu_{r}^{+}-\nu_{r}^{-}, r=1, \cdots, k$, are the Jordan decompositions of the measures $\mu_{r}=\mu_{r}^{+}+\mu_{r}^{-}$, i.e., $\nu_{r}^{+}=\mu_{r}^{+}, \nu_{r}^{-}=\mu_{r}^{-}$. Since the measures $\nu_{r}$ are absolutely continuous with respect to $\mu$, the Radon-Nikodym derivatives $\theta_{r}=d \nu_{r} / d \mu, r=1, \cdots, k$, exist $\mu$-almost everywhere in $A$, and it is proved that $\|\theta\|=1, \theta=\left(\theta_{1}, \cdots, \theta_{k}\right)$, $\mu$-almost everywhere in $A$ ( $\$ 5)$. Finally, it is proved ( $\$ 6)$ that the integral $\Im$ admits of the following integral representation

$$
\Im(f, T, \phi)=\int_{A} f[p(w), \theta(w)] d \mu .
$$

The present paper extends to all integrals $\Im$ results proved in [3] for the analogous integrals of surface area theory.

Presented to the Society, January 21, 1959 under the title The problem of extension of quasi additive set functions; received by the editors February 23, 1961.

(1) This research has been supported by ARDC, project AF 18(600)-1484. 
1. Quasi additive set functions. Let $A$ be a set, $\{I\}$ a collection of subsets $I$ of $A$ which we will denote as "intervals," (B) a collection of subsets $G$ of $A$ which we will denote as "open" sets of $A$. We shall suppose that $A \in(5)$.

Let $\mathfrak{D}=\{D\}$ be a family of finite systems $D$ of sets $I \in\{I\}$, i.e., $D=[I]$ $=\left[I_{1}, \cdots, I_{n}\right]$. For any nonempty set $G \in \mathcal{S}$ and $D=[I] \in \mathfrak{D}$, let $D_{G}$ denote the subset of all $I \in D$ which are completely contained in $G$; i.e., $D_{G}$ $=[I, I \in D, I \subset G]$. Finally, let $\mathfrak{D}_{G}$ denote the collection of all $D_{G}$ obtained by systems $D \in \mathfrak{D}$. We shall suppose that

$\left(\mathrm{b}_{1}\right)$ either $\left(\mathrm{b}_{1}^{\prime}\right)$ any two sets $I, J \in D_{G}, D_{G} \in \mathfrak{D}_{G}$, are disjoint, or $\left(\mathrm{b}_{1}^{\prime \prime}\right) A$ is a topological space and any two sets $I, J \in D_{G}, D_{G} \in \mathcal{D}_{G}$, are nonoverlapping;

$\left(b_{2}\right)$ For every nonempty $G \in(S)$ the collection $\mathfrak{D}_{a}$ is nonempty; i.e., there are systems $D \in \mathcal{D}, D=[I]$, such that $I \subset G$ for some $I \in D$.

Let $\delta\left(D_{G}, G\right)$ be a function (mesh) defined for every $D_{G} \in \mathcal{D}_{G}$ and $G \in \mathbb{S}$ satisfying the following axioms:

$\left(\mathrm{d}_{1}\right) 0<\delta\left(D_{G}, G\right)<\infty$ for every $D_{G} \in \mathfrak{D}_{G}$;

$\left(\mathrm{d}_{2}\right)$ given $\epsilon>0$ and $G \in \mathbb{G}, G \neq \varnothing$, there are systems $D_{G} \in \mathfrak{D}_{G}$ with $0<\delta\left(D_{G}, G\right)<\epsilon$

$\left(\mathrm{d}_{3}\right)$ given $\tau>0$ and any nonempty $G \in \Theta$, there is a number $\nu=\nu(\tau, G)>0$ such that, for every system $D \in \mathfrak{D}$ with $\delta(D, A)<\nu$ we have $\delta\left(D_{G}, G\right)<\tau$ and $D_{G}$ is nonempty.

Axioms $\left(b_{1}\right),\left(d_{1}\right),\left(d_{2}\right)$ are analogous to the ones proposed in [1] where $A$ was the only set $G$; axioms $\left(\mathrm{b}_{2}\right),\left(\mathrm{d}_{3}\right)$ establish a relation between the sets $G$ and $A$. For the sake of simplicity, we shall of ten denote by $D$ instead of $D_{G}$ any system $D_{G} \in \mathfrak{D}_{G}$.

Let $\phi(I)=\left(\phi_{1}, \cdots, \phi_{k}\right), I \in\{I\}$, be any real vector set function defined for every $I \in\{I\}$.

We shall say that $\phi(I), I \in\{I\}$, is quasi additive, with respect to the mesh $\delta(D, G)$ and the families $\{I\}, \mathcal{S}, \mathfrak{D}$, provided $(\phi)$ given $\epsilon>0$ and $G \in \mathbb{S}$, there is a number $\eta=\eta(\epsilon ; G)>0$ such that, if $D_{0 G}=[I]$ is any system in $\mathfrak{D}_{G}$ with $\delta\left(D_{0 G}, G\right)<\eta$, then there is also a number $\lambda=\lambda\left(\epsilon, D_{0 G}, G\right)>0$ such that, for every system $D_{G}=[J], D_{G} \in \mathfrak{D}_{G}$, with $\delta\left(D_{G}, G\right)<\lambda$, we have

$$
\begin{gathered}
\sum_{I \in D_{G}}\left\|\sum_{J \subset I} \phi(J)-\phi(I)\right\|<\epsilon, \\
\sum^{\prime}\|\phi(J)\|<\epsilon,
\end{gathered}
$$

where $\sum^{\prime}$ ranges over all $J \in D_{G}$ not completely contained in any $I \in D_{0 G}$.

We shall denote by $S\left(\phi, D_{G}\right)$ the sums

$$
S\left(\phi, D_{G}\right)=\sum_{I \in D_{G}} \phi(I)
$$


If $m$ is any real number, let $m^{+}, m^{-}$be, as usual, the numbers $m^{+}$ $=(|m|+m) / 2, m^{-}=(|m|-m) / 2$.

We shall say that a scalar vector function $\psi(I), I \in\{I\}$, is quasi subadditive provided the statement $(\psi)$ holds which is analogous to $(\phi)$ where $\left(\phi_{1}\right)$, $\left(\phi_{2}\right)$, are replaced by the single relation

$$
\sum_{I \in D_{G}}\left[\sum_{J \subset I} \psi(J)-\psi(I)\right]^{-}<\epsilon .
$$

As a consequence of $[1,3$.iv $]$ we have now

(1.i) Under hypotheses $(\phi),(\mathrm{b}),(\mathrm{d})$ and for every $G \in \mathbb{B}$ the limits exist

$$
\begin{aligned}
\mathfrak{B}(G)=\mathfrak{B}(\phi, G) & =\lim _{\delta(D G) \rightarrow 0} S\left(\phi, D_{G}\right), & \mathfrak{B}=\left(\mathfrak{B}_{1}, \cdots, \mathfrak{B}_{k}\right), \\
\mathfrak{B}_{r}(G)=\mathfrak{B}\left(\phi_{r}, G\right) & =\lim _{\delta(D G) \rightarrow 0} S\left(\phi_{r}, D_{G}\right), & -\infty<\mathfrak{B}_{r}<+\infty, \\
V(G)=V(\|\phi\|, G) & =\lim _{\delta(D G) \rightarrow 0} S\left(\|\phi\|, D_{G}\right), & 0 \leqq V \leqq+\infty, \\
V_{r}(G)=V\left(\left|\phi_{r}\right|, G\right) & =\lim _{\delta(D G) \rightarrow 0} S\left(\left|\phi_{r}\right|, D_{G}\right), & 0 \leqq V_{r} \leqq+\infty, \\
V_{r}^{+}(G)=V\left(\phi_{r}^{+}, G\right) & =\lim _{\delta(D G) \rightarrow 0} S\left(\phi_{r}^{+}, D_{G}\right), & 0 \leqq V_{r}^{+} \leqq+\infty, \\
V_{r}^{-}(G)=V\left(\phi_{r}^{-}, G\right) & =\lim _{\delta(D G) \rightarrow 0} S\left(\phi_{r}^{-}, D_{G}\right), & 0 \leqq V_{r}^{-} \leqq+\infty,
\end{aligned}
$$

where $D_{G} \in \mathfrak{D}_{G}, G \in \mathbb{S}$, and $r=1, \cdots, k$.

We shall denote by $V(\|\phi\|, G)$ the total variation of $\phi$ with respect to $G$ (or in $G$ ), while $V(\|\phi\|, A$ ) is the total variation of $\phi$ in the whole space $A$, (or simply the total variation of $\phi$ ). As a consequence of $[1,3 . v]$ we have

(1.ii) Under the same hypotheses as in (1.i), and $V(A)<+\infty$, we have

$$
\begin{aligned}
& V_{r}^{+}(G)-V_{r}^{-}(G)=\mathfrak{B}_{r}(G), \\
& V_{r}^{+}(G)+V_{r}^{-}(G)=V_{r}(G), \\
& \left|\mathfrak{B}_{r}(G)\right| \leqq V_{r}(G) \leqq V(G), \\
& \|\mathfrak{B}(G)\|=\left[\sum_{r=1}^{k} \mathfrak{B}_{r}^{2}(G)\right]^{1 / 2} \leqq\left[\sum_{r=1}^{k} V_{r}^{2}(G)\right]^{1 / 2} \leqq V(G) \leqq \sum_{r=1}^{k} V_{r}(G) .
\end{aligned}
$$

Note that the limits above are determined by means of the collection $\mathfrak{D}_{G}$ of systems $D_{G}$, and the collection $\mathfrak{D}_{G}$ is thought of as partially ordered according to $\delta\left(D_{G}, G\right)$ decreasing.

Note that, given $\tau>0$ arbitrary and any $G \in(S$, there is a number $\nu=\nu(\tau, G)>0$ with properties stated in $\left(\mathrm{d}_{3}\right)$. Thus, for any $D \in \mathbb{D}$ with $\delta(D, A)<\nu$, the corresponding system $D_{G} \in \mathfrak{D}_{G}$ is nonempty, and $\delta\left(D_{G}, G\right)<\tau$. Thus, as a consequence of $\left(d_{3}\right)$, the limits (1.i) can be determined also by means of the same collection $\mathfrak{D}_{G}$ of systems $D_{G}=[I \in D, I \subset G, D \in \mathfrak{D}]$, where 
this collection is partially ordered according to $\delta(D, A)$ decreasing. In other words, we have

(1.iii) $\mathfrak{B}(G)=\mathfrak{B}(\phi, G)=\lim _{\delta(D, A) \rightarrow 0} S\left(\phi, D_{G}\right)$, and analogous relations hold for $\phi_{r},\|\phi\|,\left|\phi_{r}\right|, \phi_{r}^{+}, \phi_{r}^{-}$.

(1.iv) If $G_{1} \subset G_{2}, G_{1}, G_{2} \in \oiint$, then $V\left(G_{1}\right) \leqq V\left(G_{2}\right)$. In particular $V(G) \leqq V(A)$ for every $G \in$ \$. A nalogous relations hold for $V_{r}, V_{r}^{+}, V_{r}^{-}$.

Proof. Take $\tau=1$ in $\left(\mathrm{d}_{3}\right)$ and let $\nu=\min \left(\nu_{1}, \nu_{2}\right), \nu_{i}=\nu\left(1, G_{i}\right), i=1,2$. Then, for all $D \in \mathfrak{D}$, with $\delta(D, A)<\nu$, the corresponding systems $D_{i}=D_{G i} \subset D, i=1,2$, are not empty and $D_{1} \subset D_{2}$. Hence, $S\left(\|\phi\|, D_{1}\right) \leqq S\left(\|\phi\|, D_{2}\right)$. As $\delta(D, A) \rightarrow 0$ the corresponding sums approach $V\left(G_{1}\right)$ and $V\left(G_{2}\right)$ respectively. Hence $V\left(G_{1}\right)$ $\leqq V\left(G_{2}\right)$. Analogously for the other relations.

As a consequence of (1.iv) we conclude that $V(A)<+\infty$ implies $V(G)$, $V_{r}(G), V_{r}^{+}(G), V_{r}^{-}(G)<+\infty$ for all $G \in(\$, r=1, \cdots, k$. As a consequence of (1.iii) and of $[1,3 . v i$, vii $]$ we have

(1.v) Under the same hypotheses as in (1.i), and $V(A)<+\infty$, all functions $\phi, \phi_{r},\|\phi\|, \phi_{r}, \phi_{r}^{+}, \phi_{r}^{-}$are quasi additive with respect to $\delta$ and $\mathfrak{D}$ in any $G \in(S$. Also, given $G \in(S$ and $\epsilon>0$, there is $a \mu=\mu(\epsilon, G)>0$ such that

$$
\left\|S\left(\phi, D_{G}\right)-\mathfrak{B}(G)\right\|<\epsilon, \quad\left|S\left(\|\phi\|, D_{G}\right)-V(G)\right|<\epsilon,
$$

for every $D_{G} \in \mathfrak{D}_{G}$ with $\delta\left(D_{G}, G\right)<\mu$, and analogous relations hold for $V_{r}$, $V_{r}^{+}, V_{r}^{-}, r=1, \cdots, k$. In addition, if $D_{0 G}, D_{0 G} \in \mathfrak{D}_{G}, \delta\left(D_{0 G}, G\right)<\mu(\epsilon, G)$, there is $a \lambda=\lambda\left(\epsilon, D_{0 G}, G\right)>0$ such that for any system $D_{G}$ with $\delta\left(D_{G}, G\right)<\lambda$ we have

$$
\begin{aligned}
\sum_{I}\left\|\sum_{(I)} \phi(J)-\phi(I)\right\|<\epsilon, \quad & \sum^{\prime}\|\phi(J)\|<\epsilon, \\
\sum_{I}\left|\sum^{(I)}\|\phi(J)\|-\|\phi(I)\|\right|<\epsilon, &
\end{aligned}
$$

where $\sum_{I}$ ranges over all $I \in D_{0 G}, \sum^{(I)}$ over all $J \in D_{G}$ with $J \subset I$, and $\sum^{\prime}$ ranges over all $J \in D_{G}, J \nsubseteq I$ for any $I \in D_{0 G}$.

2. Connections with a topology in $A$. We shall now suppose that

(a) $A$ is a topological space; $\mathfrak{U}$ is the collection of all open sets of $A$, and $\mathbb{B}$ is a subcollection of open sets of $A$ containing $A$; hence $\mathfrak{U}$ is closed with respect to infinite union and finite intersection, $\varnothing \in U, A \in U$, and (अ) $\subset \mathfrak{U}, A \in ß$ ( $\varnothing$ the empty set).

Also, from now on, under hypothesis (b) of $\S 1$ we shall understand $\left(b_{1}\right)$ and $\left(b_{2}\right)$, and the same convention is made for $(d)$ and $(\phi)$.

Suppose that all hypotheses (a), (b), (d), ( $\phi$ ) hold and, in addition, that

(c) Each set $I \in\{I\}$ is connected.

If $\Re$ is the collection of all closed sets $K \subset A$, i.e. $\Re=[K, A-K \in \mathfrak{U}]$, then the collection $\Omega$ is closed with respect to the operation of infinite intersection and finite union. The closure $\bar{M}$ of a set $M \subset A$ is the intersection of all closed sets $K \supset M$. As a consequence, if $G_{1}, G_{2} \in\left(B, G_{1} \cap G_{2}=\varnothing\right.$, then $\bar{G}_{1} \cap G_{2}=G_{1} \cap \bar{G}_{2}$ 
$=\varnothing$. Indeed $M=A-G_{2}$ is closed, $M \supset G_{1}$, and $\bar{G}_{1} \subset M$; hence $\bar{G}_{1} \cap G_{2}=\varnothing$, and for the same reason $G_{1} \cap \bar{G}_{2}=\varnothing$.

Note that if $I=\cup_{i} G_{i}, G_{i} \cap G_{j}=\varnothing, i \neq j, i, j=1,2, \cdots$, and $I \in\{I\}$, then $I \subset G_{i}$ for one and only one $i$, as a consequence of (c). Suppose indeed $I \cap G_{i}$

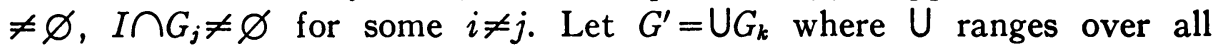
$k=1,2, \cdots, k \neq j$, and note that $G^{\prime} \in \mathfrak{U}, G_{j} \cap G^{\prime}=\varnothing, I \cap G_{j} \neq \varnothing, I \cap G^{\prime}=\varnothing$,

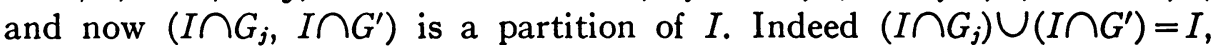
$\left(I \cap G_{j}\right) \cap\left(I \cap G^{\prime}\right)=\varnothing, \quad \mathrm{Cl}\left(I \cap G_{j}\right) \cap\left(I \cap G^{\prime}\right)=\varnothing, \quad\left(I \cap G_{j}\right) \cap \mathrm{Cl}\left(I \cap G^{\prime}\right)=\varnothing$. This contradicts (c). Hence $I \cap G_{i}=\varnothing$ for at most one $i$. Since $I \subset \cup_{i} G_{i}$, we conclude that $I \subset G_{i}$ for one and only one $i$.

(2.i) Under hypotheses (a), (b), (c), (d), ( $\phi)$, and $V(A)<+\infty$, for every sequence (finite, or countable) of sets $G_{i} \in \mathcal{B}$ with $G_{0}=\cup_{i} G_{i} \in \mathbb{G}, G_{i} \cap G_{j}=\varnothing$, $i, j=1,2, \cdots, i \neq j$, we have

$$
\mathfrak{B}\left(G_{0}\right)=\sum_{i} \mathfrak{B}\left(G_{i}\right), \quad V\left(G_{0}\right)=\sum_{i} V\left(G_{i}\right),
$$

and analogous relations hold for $V_{r}, V_{r}^{+}, V_{r}^{-}, r=1, \cdots, k$.

Proof. Suppose first that the sequence $G_{i}, i=1, \cdots, N$, is finite. Let us consider the numbers $\nu_{i}=\nu\left(1, G_{i}\right), i=0,1, \cdots, N$, defined in $\left(\mathrm{d}_{3}\right)$, and put $\nu=\min \left[\nu_{0}, \nu_{1}, \cdots, \nu_{N}\right]$. Then, for every $D \in \mathfrak{D}$ with $\delta(D, A)<\nu$, the corresponding systems $D_{i}=D_{G_{i}} \subset D, i=1, \cdots, N$, are nonempty, disjoint, and $D_{0}=D_{1} \cup D_{2} \cup \ldots \cup D_{N}$. Hence

$$
S\left(\phi, D_{0}\right)=\sum_{i=1}^{N} S\left(\phi, D_{i}\right) .
$$

By (1.iii) as $\delta(D, A) \rightarrow 0$, we obtain

$$
\mathfrak{B}\left(G_{0}\right)=\sum_{i=1}^{N} \mathfrak{B}\left(G_{i}\right) .
$$

Analogous reasoning holds for $V, V_{r}, V_{r}^{+}, V_{r}^{-}, r=1, \cdots, k$.

Suppose now that the sequence $G_{i}, i=1,2, \cdots$, is infinite. Given $\epsilon>0$, let $\mu=\mu\left(\epsilon, G_{0}\right)$ be the number defined in (1.v). Let $\nu=\nu\left(\mu, G_{0}\right)$. If $D$ is any system $D=[I] \in \mathfrak{D}$ with $\delta(D, A)<\nu$, then, for the corresponding system $D_{0}=D_{a_{0}} \subset D$ we have

$$
\begin{gathered}
\delta\left(D_{0}, G_{0}\right)<\mu, \\
\left\|\mathfrak{B}\left(G_{0}\right)-\sum_{I \subset G_{0}} \phi(I)\right\|<\epsilon, \quad\left|V\left(G_{0}\right)-\sum_{I \subset G_{0}}\|\phi(I)\|\right|<\epsilon,
\end{gathered}
$$

and analogous relations hold for $V_{r}, V_{r}^{+}, V_{r}^{-}$. If we denote by $D, D_{0}$ also the set covered by all $I \in D$, or $I \in D_{0}$, we have $D_{0} \subset G_{0}=\cup_{i} G_{i}$. Since each $I \in D_{0}$ 
belongs to a well determined set $G_{i}$, and $D_{0}$ is finite, we have $D_{0} \subset G_{1} \cup G_{2} \cup \ldots$. $\cup G_{N}$ for a well determined minimum $N \geqq 1$.

Let $\lambda=\lambda\left(\epsilon, D_{0}, G_{0}\right)>0$ be the number defined in (1.v) and, for any $n \geqq N$, let $\mu_{i}=\mu\left(\epsilon / n, G_{i}\right), i=1, \cdots, n$. Let $\nu_{0}=\nu\left(\lambda, G_{0}\right), \nu_{i}=\nu\left(\mu_{i}, G_{i}\right), i=1, \cdots, n$, and $\nu_{n}^{*}=\min \left[\nu, \nu_{0}, \nu_{1}, \cdots, \nu_{n}\right]$. If $D^{\prime}=[J]$ is any system $D^{\prime} \in \mathfrak{D}$ with $\delta\left(D^{\prime}, A\right)<\nu_{n}^{*}$, then for the corresponding systems $D_{0}^{\prime}=D_{G_{0}}^{\prime} \subset D^{\prime}, D_{i}^{\prime}=D_{G_{i}}^{\prime}$ $\subset D^{\prime}$, we have $D_{0}^{\prime} \subset G_{0}, D_{i}^{\prime} \subset G_{i}, \delta\left(D_{0}^{\prime}, G_{0}\right)<\lambda, \delta\left(D_{i}^{\prime}, G_{i}\right)<\mu_{i}, i=1, \cdots, n$, and each $J \in D_{0}^{\prime}$ belongs to one and only one $G_{i}, i=1, \cdots, n^{\prime}, n^{\prime} \geqq n$, and hence to $D_{0}^{\prime}=D_{1}^{\prime} \cup D_{2}^{\prime} \cup \ldots \cup D_{n}^{\prime}$. In addition, we have

$$
\begin{array}{rr}
\sum_{I \subset G_{0}}\left\|\sum_{J \in I} \phi(J)-\phi(I)\right\|<\epsilon, \quad \sum_{J \in G_{0}: J \Phi I}\|\phi(J)\|<\epsilon, \\
\left\|\mathfrak{B}\left(G_{i}\right)-\sum_{J \subset \cdot G_{i}} \phi(J)\right\|<\epsilon / n, & i=1, \cdots, n,
\end{array}
$$

and finally

$$
\begin{aligned}
\mathfrak{B}\left(G_{0}\right)-\sum_{i=1}^{n} \mathfrak{B}\left(G_{i}\right)= & \left\{\mathfrak{B}\left(G_{0}\right)-\sum_{I \subset G_{0}} \phi(I)\right\}-\sum_{I \subset G_{0}}\left[\sum_{J \subset I} \phi(J)-\phi(I)\right] \\
& -\sum^{*} \phi(J)+\sum_{i=1}^{n}\left[\sum_{J \subset G_{i}} \phi(J)-\mathfrak{B}\left(G_{i}\right)\right],
\end{aligned}
$$

where $\sum^{*}$ ranges over all $J \in D_{0}^{\prime}$ with $J \subset G_{0}, J \nsubseteq I$ for any $I \in D, J \subset G_{i}$ for some $1 \leqq i \leqq n$. Thus $\sum^{*}$ is less inclusive than the second sum in (2.2). By virtue of (2.1) and (2.2), (2.3), we have

$$
\left\|\mathfrak{B}\left(G_{0}\right)-\sum_{i=1}^{n} \mathfrak{B}\left(G_{1}\right)\right\|<4 \epsilon
$$

for all $n \geqq N$. Thus, the series below is convergent and

$$
\mathfrak{B}\left(G_{0}\right)=\sum_{i=1}^{\infty} \mathfrak{B}\left(G_{i}\right) \text {. }
$$

Analogous proof holds for $V$. Hence

$$
V\left(G_{0}\right)=\sum_{i=1}^{\infty} V\left(G_{i}\right)
$$

where this series is convergent, and hence the previous one is absolutely convergent. The same reasoning holds for $V_{r}, V_{r}^{+}, V_{r}^{-}, r=1,2, \cdots, k$.

We shall now consider the following requirements:

$\left(\mathrm{H}_{1}\right)$ If $G_{i} \in \mathbb{S}, i=1,2, \cdots$, and $G_{i} \rightarrow \varnothing$ as $i \rightarrow \infty$, then $V\left(G_{i}\right) \rightarrow 0$ as $i \rightarrow \infty$, and analogous relations hold for $\mathfrak{B}, V_{r}, V_{r}^{+}, V_{r}^{-}$. 
$\left(\mathrm{H}_{2}\right)$ If $G_{0}, G_{i} \in(\$), i=1,2, \cdots, G_{i} \subset G_{i+1}, G_{i} \rightarrow G_{0}$ as $i \rightarrow \infty$, then $V\left(G_{i}\right)$ $\rightarrow V\left(G_{0}\right)$ as $i \rightarrow \infty$, and analogous relations hold for $\mathfrak{B}, V_{r}, V_{r}^{+}, V_{r}^{-}$.

$\left(\mathrm{H}_{3}\right)$ If $G_{i} \in \$, i=1,2, \cdots$, and $G=\cup_{i} G_{i} \in \$, G_{1} \cup \cdots \cup G_{n} \in \$$ for all $n$, then $V(G) \leqq \sum_{i} V\left(G_{i}\right)$, and analogous relations hold for $V_{r}, V_{r}^{+}, V_{r}^{-}$.

Neither of these requirements is a consequence of the quasi additivity of the function $\phi$ and of the general hypotheses. This can be seen by examples.

Suppose first $A=(0<u<1),\{I\}$ the collection of all open subintervals of $A$, say $I=(a<u<b), 0 \leqq a<b \leqq 1$, D the family of all finite systems $D=[I]$ of nonoverlapping intervals $I$, \& the collection of all open subsets $G$ of $A$. Now suppose $\phi$ a scalar, $\phi(I)=1$ if $I=(0, b), 0<b \leqq 1$, and $\phi(I)=0$ if $I=(a, b)$, $0<a<b \leqq 1$. A mesh $\delta\left(D_{G}, G\right)$ for $D_{G}=[I], I=(a, b) \subset G$, can be defined as follows. If $G$ contains no interval $(0, b), b>0$, then we take $\delta\left(D_{G}, G\right)$ $=\max (b-a)$. If both $G$ and $D_{G}$ contain intervals $(0, b),\left(0, b^{\prime}\right)$ respectively, $0<b^{\prime} \leqq b$, then we take again $\delta\left(D_{G}, G\right)=\max (b-a)$. If $G$ contains an interval $(0, b), b>0$, and $D_{G}$ contains no interval $\left(0, b^{\prime}\right), 0<b^{\prime} \leqq b$, then we take $\delta\left(D_{G}, G\right)=1+\max (b-a)$. Obviously, $\delta$ is a mesh satisfying axioms (d), $\phi$ is quasi additive with respect to $\delta,\{I\},(B), \mathfrak{D}$, and requirements (a), (b), (c) are satisfied. Now we have $\|\phi\|=\phi, V(\phi, A)=1, V(\phi, G)=1$ if $G$ contains an interval $(0, b), b>0$, and $V(\phi, G)=0$ otherwise. If we consider the sequence $G_{i}=(0,1 / i), i=1,2, \cdots$, we have $V\left(\phi, G_{i}\right)=1$ for all $i$, though $\lim G_{i}=\varnothing$. Thus $\left(\mathrm{H}_{1}\right)$ is not satisfied. If we consider the sequence $G_{i}=(1 / i, 1)$, we have $G=\mathrm{U}_{i} G_{i}=(0,1)$, and $V(\phi, G)=1, V\left(\phi, G_{i}\right)=0$ for all $i$. Thus $\left(\mathrm{H}_{3}\right)$ is not satisfied. We have also $G_{i} \subset G_{i+1}, G_{i} \rightarrow G$, and thus $\left(\mathrm{H}_{2}\right)$ is not satisfied.

As a second example let us consider the one given in [2, Note, p. 400]. There, $A$ is the closed unit square $A \subset E_{2},\{I\}$ is the set of all simple closed polygonal regions in $A, \mathfrak{D}$ the collection of all finite systems $D=[I]$ of nonoverlapping $I \in\{I\}, \&$ the collection of all sets $G \subset A$ which are open in $A$, $\phi(I)$ is the scalar function $u(I)$ representing the signed area of the (flat) continuous mapping $T: A \rightarrow E_{2}^{\prime}$ defined there. For any set $G \in(S)$ and finite system $D=[I]$ of nonoverlapping closed simple polygonal regions $I \subset G$, let $d, m, \mu$ be the indices of $D$ with respect to the mapping $(T, G)$ defined in [2, p. 358], then $\delta=d+m+\mu$ is a mesh satisfying axioms (d), and $\phi$ is quasi additive with respect to $\delta,\{I\}, \mathfrak{S}, \mathfrak{D}$, as mentioned in [1, $\$ 4$, Example 12]. Now we have $\|\phi\|=|\phi|$, and $V$ is the Geöcze area of the mapping $T$. As shown in [2, Note, p. 400], $\left(\mathrm{H}_{3}\right)$ is not satisfied (not even for a system of two sets $\left.G_{i}\right)$.

The following further axiom would allow us to state $\left(\mathrm{H}_{3}\right)$ as a theorem (see 2.iii below) :

(e) Given any two distinct sets $G_{1}, G_{2} \in\left(\$, G_{1} \cap G_{2} \neq \varnothing\right.$, and $G_{0}=G_{1} \cup G_{2}$, and any $I=\{I\}, I \subset G_{0}$, with $I \cap G_{1}=\varnothing, I \cap G_{2}=\varnothing$, there is a number 
$\chi=\chi\left(I, G_{1}, G_{2}\right)>0$ such that for any system $D_{G_{0}}=[J], D_{G_{0}} \in \mathscr{D}_{a_{0}}$ with $\delta\left(D_{G_{0}}, G_{0}\right)<\chi$, and for any $J \in D_{G_{0}}$ with $J \subset I$ we have either $J \subset G_{1}$, or $J \subset G_{2}$, or both $\left.{ }^{2}\right)$.

(2.ii) The hypotheses (a), (b), (c), (d), (e), ( $\phi)$, and $V(A)<\infty$ imply $V(G)$ $\leqq V\left(G_{1}\right)+\cdots+V\left(G_{N}\right)$ for all $G_{i} \in \$, i=1, \cdots, N$, with $G_{1} \cup \cdots \cup G_{n} \in \oiint$ for all $n=1, \cdots, N, G=\bigcup_{i} G_{i}$, and analogous relations hold for $V_{r}, V_{r}^{+}, V_{r}^{-}$.

Proof. Let us suppose first that we have two sets $G_{1}, G_{2} \in ß$ with $G_{1} \cap G_{2}$ $\neq \varnothing$, and $G_{0}=G_{1} \cup G_{2} \in\left(S\right.$. Given $\epsilon>0$ let us determine the numbers $\mu_{j}=\mu\left(\epsilon, G_{j}\right)$ of (1.v), $j=0,1,2$. Then let us determine the numbers $\nu_{j}=\nu\left(\mu_{j}, G_{j}\right), j=0,1,2$, of $\left(\mathrm{d}_{3}\right)$. Finally, let $D_{0}=[I]$ be any system $D_{0} \in \mathfrak{D}$ such that $\delta\left(D_{0}, A\right)$ $<\min \left[\nu_{j}, j=0,1,2\right]$. Then the corresponding systems $D_{0 j} \equiv D_{0 G} \subset D_{0}$ of all $I \in D_{0}$ with $I \subset G_{j}, j=0,1,2$, satisfy the relations

$$
\begin{aligned}
\delta\left(D_{0 j}, G_{j}\right) & <\mu_{j}, \\
\left|V\left(G_{j}\right)-\sum_{I \subset G_{j}}\|\phi(I)\|\right| & <\epsilon, \quad j=0,1,2 .
\end{aligned}
$$

Now let us determine the numbers $\lambda_{j}=\lambda\left(\epsilon, D_{0 j}, G_{j}\right)$ of (1.v) and the corresponding numbers $\nu_{j}^{\prime \prime}=\nu\left(\lambda_{j}, G_{j}\right), j=0,1,2$, of $\left(\mathrm{d}_{3}\right)$. Also, for every $I \subset D_{00}$ (i.e., $I \subset G_{0}, I \in D_{0}$ ), with $I \cap G_{1} \neq \varnothing, I \cap G_{2} \neq \varnothing$ (if any), let us determine the number $\chi_{I}=\chi\left(I, G_{1}, G_{2}\right)$ of (e). Let $\chi=\min \chi_{I}$ for all $I$ as above, if this class is not empty; otherwise put $\chi=1$. Finally, let us determine the number $\nu^{\prime \prime \prime}=\nu\left(\chi, G_{0}\right)$ of $\left(\mathrm{d}_{3}\right)$.

Let $D=[J]$ be any system $D \in \mathfrak{D}$ with $\delta(D, A)<\min \left[\nu_{j}, \nu_{j}^{\prime \prime}, \nu^{\prime \prime \prime}, j=0,1,2\right]$. Then the corresponding systems $D_{j}=D_{G_{j}} \subset D$ of all $J \in D$ with $J \subset G_{j}$, $j=0,1,2$, satisfy the relations:

$$
\begin{aligned}
\delta\left(D_{j}, G_{j}\right)<\lambda_{j}, \mu_{j}, \quad \delta\left(D_{0}, G_{0}\right)<\chi, & \\
\sum_{I \subset G_{j}}\left|\sum_{J \subset I}\|\phi(J)\|-\|\phi(I)\|\right|<\epsilon, & j=0,1,2 .
\end{aligned}
$$

By $\delta\left(D_{G_{0}}, G_{0}\right)<\chi$ we conclude by virtue of (e) that, for every $J \in D_{G_{0}}, J \subset I$ for some $I \subset G_{0}, I \in D_{00}$, we have either $J \subset G_{1}$, or $J \subset G_{2}$, or both. Hence, the following identity holds:

(2) Statement (e) and consequent statement $\left(\mathrm{H}_{3}\right)$ are actually proved in surface area theory, when (ङ) is the collection of all subsets of $A$ which are "whole" and open in $A$ (see [2, pp. 396400, and in particular p. 399]). The same holds for $\left(\mathrm{H}_{1}\right)$ [2, p. 396], for $\left(\mathrm{H}_{2}\right)[2$, p. 120] and the other axioms. 


$$
\begin{aligned}
V\left(G_{1}\right) & +V\left(G_{2}\right)-V\left(G_{0}\right) \\
= & {\left[V\left(G_{1}\right)-\sum_{J \subset G_{1}}\|\phi(J)\|\right]+\left[V\left(G_{2}\right)-\sum_{J \subset G_{2}}\|\phi(J)\|\right] } \\
& +\sum_{J \subset G_{1} \cap G_{2} ; J_{\subset} I}\|\phi(J)\|+\sum_{J \subset G_{1 ;} ; J_{\phi} I}\|\phi(J)\|+\sum_{J \subset G_{2 ;} J_{\Phi} I}\|\phi(J)\| \\
& +\sum_{I \subset G_{0}}\left[\sum_{J \subset I}\|\phi(J)\|-\|\phi(I)\|\right]+\left[\sum_{I \subset G_{0}}\|\phi(I)\|-V\left(G_{0}\right)\right] \\
= & m_{1}+m_{2}+\cdots+m_{7} .
\end{aligned}
$$

By (2.4), (2.6) we have $m_{1}, m_{2}, m_{7} \geqq-\epsilon$, and obviously $m_{3}, m_{4}, m_{5} \geqq 0$. By (2.5) we have $m_{0} \geqq-\epsilon$. Thus $V\left(G_{1}\right)+V\left(G_{2}\right)-V\left(G_{0}\right) \geqq-7 \epsilon$ where $\epsilon>0$ is arbitrary. Thus

$$
V\left(G_{1}\right)+V\left(G_{2}\right)-V\left(G_{0}\right) \geqq 0,
$$

for all $G_{1}, G_{2} \in \mathcal{S}, G_{0}=G_{1} \cup G_{2} \in \mathcal{S}$, with $G_{1} \cap G_{2} \neq \varnothing$. By (2.i) this relation holds also if $G_{1} \cap G_{2}=\varnothing$. Thus

$$
V\left(G_{1} \cup G_{2}\right) \leqq V\left(G_{1}\right)+V\left(G_{2}\right)
$$

for all $G_{1}, G_{2} \in$ S, with $G_{1} \cup G_{2} \in$ S.

If $G_{1}, \cdots, G_{N} \in(S)$ are given sets, as in (2.ii), $2 \leqq N<+\infty$, then by applying (2.8) $N-1$ times to the pairs $\left(G_{1}, G_{2}\right),\left(G_{1} \cup G_{2}, G_{3}\right), \cdots$, $\left(G_{1} \cup \ldots \cup G_{N-1}, G_{N}\right)$, we conclude that

$$
V\left(G_{1} \cup G_{2} \cup \cdots \cup G_{N}\right) \leqq V\left(G_{1}\right)+\cdots+V\left(G_{N}\right)
$$

for all $G_{1}, \cdots, G_{N} \in(\$, 2 \leqq N<\infty$, as in (2.ii). The corresponding relations for $V_{r}, V_{r}^{+}, V_{r}^{-}$follow by analogous argument.

(2.iii) The hypotheses (a), (b), (c), (d), (e), ( $),\left(\mathrm{H}_{2}\right)$, and $V(A)<+\infty$, imply $\left(\mathrm{H}_{3}\right)$.

Let $G_{i}, i=1,2, \cdots$, be any sequence of sets $G_{i} \in \$$, with $H_{n}=\cup_{i=1}^{n} G_{i} \in$ S, $n=1,2, \cdots, G_{0}=\sum_{i=1}^{\infty} G_{i}, G_{0} \in \circlearrowleft$. Then we have $H_{n} \subset H_{n+1}, H_{n} \rightarrow G_{0}$ as $n \rightarrow \infty$. Thus, by $\left(\mathrm{H}_{2}\right)$, we have $V\left(H_{n}\right) \rightarrow V\left(G_{0}\right)$ as $n \rightarrow \infty$ and, given $\epsilon>0$ there is an $n_{0}$ such that $0 \leqq V\left(G_{0}\right)-V\left(H_{n}\right)<\epsilon$ for all $n \geqq n_{0}$. On the other hand we have, by the considerations above, $V\left(H_{n}\right) \leqq V\left(G_{1}\right)+\cdots+V\left(G_{n}\right)$ and $V\left(G_{i}\right) \geqq 0, i=1,2, \cdots$. Thus

$$
\begin{aligned}
V\left(G_{0}\right) & \leqq V\left(H_{n}\right)+\epsilon \leqq V\left(G_{1}\right)+\cdots+V\left(G_{n}\right)+\epsilon \\
& \leqq \sum_{i=1}^{\infty} V\left(G_{i}\right)+\epsilon
\end{aligned}
$$

for every $\epsilon>0$, and hence 


$$
V\left(G_{0}\right) \leqq \sum_{i=1}^{\infty} V\left(G_{i}\right)
$$

The corresponding relations for $V_{r}, V_{r}^{+}, V_{r}^{-}$follow by an analogous argument. Thereby (2.iii) is proved.

The following further axiom would allow us to state $\left(\mathrm{H}_{2}\right)$ as a theorem.

(g) The sets $I \in\{I\}$ are compact (in the topological space $A$ with topology $\mathfrak{u}$ ).

(2.iv) The hypotheses (a), (b), (c), (d), (e), (g), ( $\phi)$, and $V(A)<+\infty$, imply $\left(\mathrm{H}_{2}\right)$.

Proof. Given $\epsilon>0$, let us determine the number $\mu=\mu\left(\epsilon, G_{0}\right)$ of (1.v). Then let us determine the number $\nu=\nu\left(\mu, G_{0}\right)$ of $\left(\mathrm{d}_{3}\right)$. Let $D_{0}=[I]$ be any system $D_{0} \in \mathfrak{D}$ such that $\delta\left(D_{0}, A\right)<\nu$. Then the corresponding system $D_{00} \subset D_{0}$ of all $I \in D_{0}$ with $I \subset G_{0}$ satisfies the relations

$$
\delta\left(D_{00}, G_{0}\right)<\mu, \quad\left|V\left(G_{0}\right)-\sum_{I \subset G_{0}}\|\phi(I)\|\right|<\epsilon,
$$

and analogous relations for $\mathfrak{B}, V_{r}, V_{r}^{+}, V_{r}^{-}$.

Since the set $D_{00}$ covered by the sets $I \in D_{00}, I \subset G_{0}$ is compact and $D_{00} \subset G_{0}$, while $G_{i} \rightarrow G_{0}$ as $i \rightarrow \infty$, there is an $n$ such that $D_{00} \subset G_{i}$ for all $i \geqq n$. Thus the set $D_{0 i}$ of all $I \in D_{0}$ with $I \subset G_{i}$ coincides with $D_{00}$, say $D_{0 i} \equiv D_{00}$. For any fixed $i \geqq n$, let us consider the numbers $\mu_{i}=\mu\left(\epsilon, G_{i}\right)$, and put $\nu_{i}=\nu\left(\mu_{i}, G_{i}\right)$. Let $\lambda=\lambda\left(\epsilon, D_{00}, G_{0}\right), \lambda_{i}=\lambda\left(\epsilon, D_{0 i}, G_{i}\right)$ be the numbers defined in (1.v), and put $\sigma=\nu\left(\lambda, G_{0}\right), \sigma_{i}=\nu\left(\lambda_{i}, G_{i}\right)$. Finally, let $D=[J]$ be any system $D \in \mathscr{D}$ with $\delta(D, A)<\min \left[\nu, \nu_{i}, \sigma, \sigma_{i}\right]$. Then the corresponding system $D_{i} \subset D$ of all $J \in D$ with $J \subset G_{i}$ satisfies the relations $\delta\left(D_{i}, G_{i}\right)<\mu_{i}, \lambda_{i}$, and

$$
\begin{array}{rc}
\left|V\left(G_{0}\right)-\sum_{J \subset G_{0}}\|\phi(J)\|\right|<\epsilon, & \left|V\left(G_{i}\right)-\sum_{J \subset G_{i}}\|\phi(J)\|\right|<\epsilon, \\
\sum_{I \subset G_{0}}\left|\sum_{J \subset I}\|\phi(J)\|-\|\phi(I)\|\right|<\epsilon, & \sum_{J \subset G_{0} ; J \Phi I}\|\phi(J)\|<\epsilon, \\
\sum_{I \subset G_{i}}\left|\sum_{J \subset I}\|\phi(J)\|-\|\phi(I)\|\right|<\epsilon, & \sum_{J \subset G_{i} ; J_{\Phi} I}\|\phi(J)\|<\epsilon .
\end{array}
$$

Note that the first sum in the third line is equal to the first sum in the second line, and the second sum in the third line is $\leqq$ the second sum in the second line. Analogous relations hold for $\phi, \phi_{r},\left|\phi_{r}\right|, \phi_{r}^{+}, \phi_{r}^{-}, r=1, \cdots, k$. Note that, as a consequence, we have also

$$
\sum_{J \subset G_{0} ; J \ddagger G_{i}}\|\phi(J)\|<\epsilon, \quad\left\|\sum_{J \subset G_{0} ; J \ddagger G_{i}} \phi(J)\right\|<\epsilon, \text { etc. }
$$

Since 


$$
\begin{aligned}
V\left(G_{0}\right)-V\left(G_{i}\right)= & {\left[V\left(G_{0}\right)-\sum_{J \subset G_{0}}\|\phi(J)\|\right]-\left[V\left(G_{i}\right)-\sum_{J \subset G_{i}}\|\phi(J)\|\right] } \\
& +\sum_{J \subset G_{0} ; J_{\Phi} G_{i}}\|\phi(J)\|,
\end{aligned}
$$

by (2.11) we have also

$$
0 \leqq V\left(G_{0}\right)-V\left(G_{i}\right) \leqq \epsilon+\epsilon+\epsilon=3 \epsilon .
$$

This relation holds for all $i \geqq n$, and this proves that $V\left(G_{i}\right) \rightarrow V\left(G_{0}\right)$ as $i \rightarrow \infty$.

3. A measure $\mu$ associated to $\phi$. We recall first a few definitions. A collection $\mathfrak{A}$ of subsets $E$ of $A$ is said to be a ring if $E, F \subset \mathfrak{A}$ implies $E \cup F$, $E-F \in \mathfrak{A}$; an algebra if $\mathfrak{A}$ is a ring and $A \in \mathfrak{A}$; a $\sigma$-ring ( $\sigma$-algebra) if $\mathfrak{A}$ is a ring (algebra) and $E_{i} \in \mathfrak{A}, i=1,2, \cdots$, implies $\bigcup_{i} E_{i} \in \mathfrak{A}$. A $\sigma$-ring ( $\sigma$-algebra) is closed with respect to the operations of countable union and countable intersection $[4$, p. 24]. A ring contains the empty set $\varnothing$.

A collection $\mathfrak{A}$ of subsets $E$ of $A$ is said to be hereditary if $E \subset F, F \in \mathfrak{A}$ implies $E \in \mathfrak{A}$. A real-valued set function $m(E), E \in \mathfrak{A}$, defined on every set of a collection $\mathfrak{A}$ of subsets of $A$ is said to be monotone if $E \subset F, E, F \in \mathfrak{A}$, imply $m(E) \leqq m(F)$, is said to be countably subadditive if $E_{i} \in \mathfrak{A}, i=1,2$, ..., $\cup_{i} E_{i} \in A$ imply $m\left(\bigcup_{i} E_{i}\right) \leqq \sum_{i} m\left(E_{i}\right)$.

A set function defined on $\mathfrak{A}$ is said to be an outer measure provided $\mathfrak{A}$ is a hereditary $\sigma$-ring, if $m(\varnothing)=0$, and $m$ is real-valued, nonnegative, monotone, and countably subadditive.

We shall now suppose that a hypothesis stronger than (a) holds, namely:

(a') $A$ is a topological space, $\mathfrak{U}$ is the collection of all open sets of $A$, (B) is a subcollection of $\mathfrak{U}$ which is also closed with respect to the operations of infinite union and finite intersection, and $\varnothing \in(S, A \in \$$.

We suppose, as in $\$ 2$, that also hypotheses (b), (d), (c), ( $\phi)$ hold. Note that now $(S)$ defines a topology in $A, \mathscr{B} \subset \mathfrak{U}$. Let $\mathfrak{B}$ be the minimal $\sigma$-algebra containing $\mathfrak{G}$, and $\mathfrak{M}$ the hereditary $\sigma$-algebra of all subsets $M$ of $A$. Thus (S) $\subset \mathfrak{B} \subset \mathfrak{M}$.

For every set $M \in \mathfrak{M}$ we define the following nonnegative set functions

$$
\begin{aligned}
& \mu(M)=\operatorname{Inf}_{G \supset M} V(G), \quad \mu_{r}(M)=\operatorname{Inf}_{G \supset M} V_{r}(G), \\
& \mu_{r}^{+}(M)=\operatorname{Inf}_{G \supset M} V_{r}^{+}(G), \quad \mu_{r}^{-}(M)=\operatorname{Inf}_{G \supset M} V_{r}^{-}(G), \quad r=1, \cdots, k,
\end{aligned}
$$

where, in each relation, the infimum is taken with respect to all $G \supset M, G \in(S$. If $V(A)<+\infty$, then $\mu, \mu_{r}, \mu_{r}^{+}, \mu_{r}^{-}<+\infty$ for all $M \in \mathbb{M}$, and we can define the following real-valued set functions:

$$
\nu_{r}(M)=\mu_{r}^{+}(M)-\mu_{r}^{-}(M), \quad r=1, \cdots, k .
$$


We shall denote by $\nu(M)$ the vector set function

$$
\nu(M)=\left[\nu_{1}(M), \cdots, \nu_{k}(M)\right] .
$$

(3.i) Under the hypotheses ( $\left.\mathrm{a}^{\prime}\right),(\mathrm{b}),(\mathrm{c}),(\mathrm{d}),(\phi)$, and for every set $M \in \mathfrak{M}$, there is a sequence $G_{i}, i=1,2, \cdots$, of sets $G_{i} \in \mathbb{S}, G_{i} \supset M, i=1,2, \cdots$, such that $V\left(G_{i}\right) \rightarrow \mu(M), V_{r}\left(G_{i}\right) \rightarrow \mu_{r}(M), V_{r}^{+}\left(G_{i}\right) \rightarrow \mu_{r}^{+}(M), V_{r}^{-}\left(G_{i}\right) \rightarrow \mu_{r}^{-}(M)$ as $i \rightarrow \infty$, and, if $V(A)<+\infty$, also $\mathfrak{B}_{r}\left(G_{i}\right) \rightarrow \nu_{r}(M), r=1, \cdots, k$.

Proof. Note that if $G_{i}, G_{i}^{\prime}, i=1,2, \cdots$, are sequences of sets $G_{i}, G_{i}^{\prime} \in$ (s) and $M \subset G_{i}^{\prime} \subset G_{i}, V\left(G_{i}\right) \rightarrow \mu(M)$, then also $V\left(G_{i}^{\prime}\right) \rightarrow \mu(M)$, as a consequence of $\mu(M) \leqq V\left(G_{i}^{\prime}\right) \leqq V\left(G_{i}\right)$ (1.iv). The same holds for the other functions $\mu_{r}, \mu_{r}^{+}$, $\mu_{r}^{-}, r=1, \cdots, k$. Now, if $G_{0 i}, G_{r i}, G_{r i}^{+}, G_{r i}^{-}, i=1,2, \cdots$, are sequences of sets of $\$$ all containing $M$ such that $V\left(G_{0 i}\right) \rightarrow \mu(M), V\left(G_{r i}\right) \rightarrow \mu_{r}(M), V\left(G_{r i}^{+}\right)$ $\rightarrow \mu_{r}^{+}(M), V\left(G_{r i}^{-}\right) \rightarrow \mu_{r}^{-}(M)$ as $i \rightarrow \infty, r=1, \cdots, k$, we have only to consider the sequence $G_{i}, i=1,2, \cdots$, defined by taking for each $i$, the set $G_{i}$ which is the intersection of the sets $G_{0 i}, G_{r i}, G_{r i}^{+}, G_{r i}^{-}, r=1, \cdots, k$.

(3.ii) Under the same hypotheses as in (3.i) and for every set $M \in \mathfrak{M}$ we have

$$
\mu_{r}(M)=\mu_{r}^{+}(M)+\mu_{r}^{-}(M), \quad r=1, \cdots, k .
$$

Proof. If $G_{i}, i=1,2, \cdots$, is the sequence defined above we have $V_{r}\left(G_{i}\right)$ $=V_{r}^{+}\left(G_{1}\right)+V_{r}^{-}\left(G_{i}\right), i=1,2, \cdots$, (1.ii). As $i \rightarrow \infty$, we obtain (3.3).

(3.iii) Under the same hypotheses as in (3.i) and $V(A)<+\infty$, for every $M \in \mathfrak{M}$, we have

$$
\begin{aligned}
&\left|\nu_{r}(M)\right| \leqq \mu_{r}(M) \leqq \mu(M), r=1, \cdots, k, \\
&\|\nu(M)\|=\left[\sum_{r=1}^{k} \nu_{r}^{2}(M)\right]^{1 / 2} \leqq\left[\sum_{r=1}^{k}{ }_{\mu_{r}}^{2}(M)\right]^{1 / 2} \leqq \mu(M) \leqq \sum_{r=1}^{k} \mu_{r}(M) .
\end{aligned}
$$

This statement is a consequence of (1.ii) and (3.i), (3.2), (3.3).

(3.iv) Under the same hypotheses as in (3.i) and for every $G \in B$ we have $\mu(G)=V(G)$, and analogous relations hold for $\mu_{r}, \mu_{r}^{+}, \mu_{r}^{-}, r=1, \cdots, k$. If $V(A)<+\infty$ we have also $\nu(G)=\mathfrak{B}(G)$.

Indeed for every $U \supset G, U \in($, we have $V(U) \geqq V(G)$, and thus $V(G)$ is the minimum of $V(U)$ for all $U \supset G, U \in \mathbb{S}$.

(3.v) Under the hypotheses $\left(\mathrm{a}^{\prime}\right),(\mathrm{b}),(\mathrm{c}),(\mathrm{d}),(\phi)$, and $\left(\mathrm{H}_{1}\right)$ we have $\mu(\varnothing)$ $=\mu_{r}(\varnothing)=\mu_{r}^{+}(\varnothing)=\mu_{r}^{-}(\varnothing)=0, r=1,2, \cdots, k$, where $\varnothing$ is the empty set.

This statement is a consequence of $\left(\mathrm{H}_{1}\right)$.

(3.vi) Under the hypotheses ( $\left.\mathrm{a}^{\prime}\right),(\mathrm{b}),(\mathrm{c}),(\mathrm{d}),(\phi),(\mathrm{H})$, and $V(A)<+\infty$ the set functions $\mu, \mu_{r}, \mu_{r}^{+}, \mu_{r}^{-}$are outer measures in $\mathfrak{M}$. 
Proof. Obviously $\mu(E) \geqq 0$ for every $E \in \mathfrak{M}$, and $\mu(\varnothing)=0$. Also, $\mathfrak{M}$ is a hereditary $\sigma$-ring. If $E, F \in \mathfrak{M}, E \subset F$, then any set $G \in \mathbb{B}, G \supset F$, contains also $E$ and, if $G_{i}$ is any sequence of sets $G_{i} \in\left(B, G_{i} \supset E, i=1,2, \cdots\right.$, with $V\left(G_{i}\right) \rightarrow \mu(E)$, then we have also $G \cap G_{i} \in\left(\mathfrak{S}, E \subset G \cap G_{i} \subset G, V\left(G \cap G_{i}\right) \leqq V(G)\right.$, and hence, as $i \rightarrow+\infty$, we obtain $\mu(E) \leqq V(G)$ for every $G \in \mathbb{B}, G \supset F$. Since Inf $V(G)=\mu(F)$, we have $\mu(E) \leqq \mu(F)$, and $\mu$ is monotone. The same holds for the other functions.

If $E_{i}, i=1,2, \cdots$, is any sequence of sets $E_{i} \in \mathfrak{M}$, then we have $E_{0}$ $=\cup_{i} E_{i} \in \mathfrak{M}$. Given $\epsilon>0$, there is a set $G_{i} \in \mathbb{B}, G_{i} \supset E_{i}$, such that $\mu\left(E_{i}\right)$ $\leqq V\left(G_{i}\right) \leqq \mu\left(E_{i}\right)+\epsilon / 2^{i}$. Note that $G_{0}=\cup_{i} G_{i} \in(B), E \subset G_{0}$, and by $\left(\mathrm{H}_{3}\right)$ also

$$
\mu(E) \leqq V\left(G_{0}\right) \leqq \sum_{i=1}^{\infty} V\left(G_{i}\right) \leqq \sum_{i=1}^{\infty} \mu\left(E_{i}\right)+\epsilon,
$$

where $\epsilon>0$ is arbitrary. Hence

$$
\mu(E) \leqq \sum_{i=1}^{\infty} \mu\left(E_{i}\right),
$$

and $\mu$ is countably subadditive. Thus $\mu$ is an outer measure in $\mathfrak{M}$. Analogous reasoning holds for $\mu_{r}, \mu_{r}^{+}, \mu_{r}^{-}, r=1, \cdots, k$, and (3.vi) is proved.

As usual a set $E \in \mathfrak{M}$ is said to be $\mu$-measurable provided for every set $M \in \mathfrak{M}$ we have

$$
\mu(M)=\mu(M \cap E)+\mu(M-E) .
$$

Analogous definitions hold for all outer measures $\mu_{r}, \mu_{r}^{+}, \mu_{r}^{-}, r=1,2, \cdots, k$.

(3.vii) The collection $\mathbb{E}_{\mu}$ of all $\mu$-measurable sets $M \in \mathfrak{M}$ is a $\sigma$-ring.

This is a well-known theorem (see, e.g. [4, p. 46]). The same holds for the collections $\mathbb{E}_{\mu_{r}}, \mathfrak{E}_{\mu_{r}+}, \mathbb{E}_{\mu_{r}}$ of all $\mu_{r}, \mu_{r}^{+}, \mu_{r}^{-}$-measurable sets $M \in \mathfrak{M}$.

We need now two simple lemmas (3.viii, ix) whose proofs are given here for the sake of simplicity, though they appear in a slightly different context in $[4$, p. 45, p. 234$]$.

(3.viii) A necessary and sufficient condition for a set $E \in \mathfrak{M}$ to be $\mu$-measurable is that for every $M \in \mathfrak{R}$ we have

$$
\mu(M) \geqq \mu(M \cap E)+\mu(M-E) .
$$

Proof. Indeed $M=(M \cap E) \cup(M-E)$ and by (3.vi) also

$$
\mu(M) \leqq \mu(M \cap E)+\mu(M-E) .
$$

Thus we have

$$
\mu(M)=\mu(M \cap E)+\mu(M-E)
$$

if and only if (3.5) holds. 
(3.ix) A necessary and sufficient condition for a set $E \in \mathbb{M}$ to be $\mu$-measurable is that for every $U \in(\$)$ we have

$$
\mu(U) \geqq \mu(U \cap E)+\mu(U-E) .
$$

Proof. Suppose $E$ is $\mu$-measurable. Then

$$
\mu(M)=\mu(M \cap E)+\mu(M-E)
$$

for every $M \in \mathfrak{M}$, hence for every $M=U \in(S$, and this certainly implies (3.6). Suppose (3.6) is true for every $U \in\left(S\right.$, and take $M \in \mathfrak{M}$, and $G_{0} \in \mathbb{S}$ with $G_{0} \supset M$. Then

$$
V\left(G_{0}\right)=\mu\left(G_{0}\right) \geqq \mu\left(G_{0} \cap E\right)+\mu\left(G_{0}-E\right) \geqq \mu(M \cap E)+\mu(M-E),
$$

and, since $\mu(M)=\operatorname{Inf} V\left(G_{0}\right)$, we conclude that $\mu(M) \geqq \mu(M \cap E)+\mu(M-E)$, and this relation is proved for every $M \in \mathfrak{M}$. By (3.viii) we conclude that $E$ is measurable.

The requirement $(\mathrm{H})$ of $\S 2$, i.e., the union of axioms $\left(\mathrm{H}_{1}\right),\left(\mathrm{H}_{2}\right),\left(\mathrm{H}_{3}\right)$, is now to be replaced by a slightly stronger assumption, say $\left(\mathrm{H}^{\prime}\right)$, namely the union of $\left(\mathrm{H}_{1}\right),\left(\mathrm{H}_{2}\right),\left(\mathrm{H}_{3}\right)$, and

$\left(\mathrm{H}_{4}\right)$ Given $G_{0} \in \$$, there is a sequence $G_{i}, i=1,2, \cdots$, of sets $G_{i} \in \mathbb{B}$, such that, if $\bar{G}_{i}$ denotes the closure of $G_{i}$ in the topology $\$$, we have $G_{i} \subset G_{0}$, $G_{i} \subset \bar{G}_{i} \subset G_{i+1}$, and $V\left(G_{i}\right) \rightarrow V\left(G_{0}\right)$ as $i \rightarrow \infty$, and analogous relations hold for $\mathfrak{B}, V_{r}, V_{r}^{+}, V_{r}^{-}, r=1, \cdots, k$.

Note that the requirements alone $G_{i} \in$ S), $G_{i} \subset G_{0}, G_{i} \subset \bar{G}_{i} \subset G_{i+1}$, are trivial since the sequence $G_{i}=\varnothing, i=1,2, \cdots$, satisfy them. The following axiom, also bearing on the topology $\left(S\right.$, allows us to prove $\left(\mathrm{H}_{4}\right)$ as a theorem:

(p) Given $G_{0} \in ß$ there is a sequence $G_{i}, i=1,2, \cdots$, such that $G_{i} \in($, $G_{i} \subset G_{0}, G_{i} \subset \bar{G}_{i} \subset G_{i+1}$, and $G_{i} \rightarrow G_{0}$ as $i \rightarrow \infty$.

It is obvious that $\left(\mathrm{H}_{2}\right)$ and $(\mathrm{p})$ imply $\left(\mathrm{H}_{4}\right)$. Requirement $(\mathrm{p})$ is known in general topology (cf. $[6 ; 7 ; 8])$. The union of $\left(\mathrm{H}_{2}\right)$ and $(\mathrm{p})$ is a stronger requirement than $\left(\mathrm{H}_{4}\right)$ as $\mathrm{T}$. Nishiura [10] has proved by an example.

(3.x) Under the assumptions $\left(\mathrm{a}^{\prime}\right),(\mathrm{b}),(\mathrm{c}),(\mathrm{d}),\left(\mathrm{H}^{\prime}\right)$, and $V(A)<+\infty$, all sets $B$ of the $\sigma$-algebra $B$ are $\mu$-measurable as well as $\mu_{r}, \mu_{r}^{+}, \mu_{r}^{-}-$measurable. In other words, the restrictions of $\mu, \mu_{r}, \mu_{r}^{+}, \mu_{r}^{-}, r=1, \cdots, k$, on $\mathfrak{B}$ are all measures.

Proof. It is enough to prove that all the sets $G$ of the class (S) generating $\mathfrak{B}$ are measurable. By (3.ix) it is enough to prove that for every $U \in \mathbb{B}$ and any $G \in(S)$ we have $\mu(U) \geqq \mu(U \cap G)+\mu(U-G)$. Note that $U, U \cap G \in \mathcal{G}$, and thus we have only to prove that $V(U) \geqq V(U \cap G)+\mu(U-G)$.

By virtue of $\left(\mathrm{H}_{4}\right)$ there is a sequence of sets $G_{n} \in \Theta, n=1,2, \cdots$, such that $G_{n} \subset U \cap G, G_{n} \subset \bar{G}_{n} \subset G_{n+1}, V\left(G_{n}\right) \rightarrow V(U \cap G)$. 
Given $\epsilon>0$ and any integer $n$, let $\mu=\mu(\epsilon, U), \mu_{n}=\mu\left(\epsilon, G_{n}\right)$, and $\nu=\nu(\mu, U)$, $\nu_{n}=\nu\left(\mu_{n}, G_{n}\right)$. Let $D_{0}=[I]$ be any system $D \in \mathcal{D}$ with $\delta(D)<\min \left[\nu, \nu_{n}\right]$. Then for the corresponding systems $D_{0}=D_{U}, D_{n}=D_{G_{n}}$ we have

$$
\begin{gathered}
\delta\left(D_{0}, U\right)<\mu, \quad \delta\left(D_{n}, G_{n}\right)<\mu_{n}, \\
\left|V(U)-\sum_{I \subset U}\|\phi(I)\|\right|<\epsilon, \quad\left|V\left(G_{n}\right)-\sum_{I \subset G_{n}}\|\phi(I)\|\right|<\epsilon .
\end{gathered}
$$

Let us consider the sets $\mathrm{Cl}(U-G)$ and $\bar{G}_{n}$ where the closures are taken in the topology $\$$. On one side, if a point $w$ belongs to $\mathrm{Cl}(U-G)$, then it must be in $A-G$, i.e., $\mathrm{Cl}(U-G) \subset A-G$. On the other hand, $\bar{G}_{n} \subset U \cap G \subset G$, and hence the closed sets $\mathrm{Cl}(U-G)$ and $\bar{G}_{n}$ are disjoint. Let $W_{n} \in(S)$ be a set with $W_{n} \supset U-G, W_{n} \cap \bar{G}_{n}=\varnothing$. For instance, $A-\bar{G}_{n}$ has this property. Since $U \supset U-G$, we have also $W_{n} \cap U \supset U-G, W_{n} \cap U \cap \bar{G}_{n}=\varnothing$, where $W_{n} \cap U \in$ (S)

Let $\lambda=\lambda\left(\epsilon, D_{0}, U\right), \lambda_{n}=\lambda\left(\epsilon, D_{n}, G_{n}\right), \mu_{0}=\mu\left(\epsilon, W_{n} \cap U\right), \nu_{0}=\nu\left(\mu_{0}, W_{n} \cap U\right)$, $\nu=\nu(\lambda, U), \nu_{n}=\nu\left(\lambda_{n}, G_{n}\right)$, and let $D^{\prime}=[J]$ be any system $D^{\prime} \in \mathcal{D}$ with $\delta\left(D^{\prime}, A\right)$ $<\min \left[\nu, \nu_{n}, \nu_{0}\right]$. Then for the corresponding systems $D_{0}^{\prime}=D_{U}^{\prime}, D_{n}^{\prime}=D_{G_{n}}$, $D^{\prime}=D_{W_{n} \cap U}$, we have

$$
\begin{gathered}
\sum_{I \subset U}\left|\sum_{J \subset I}\|\phi(J)\|-\|\phi(I)\|<\epsilon, \quad \sum_{I \subset G_{n}}\right| \sum_{J \subset I}\|\phi(J)\|-\|\phi(I)\| \mid<\epsilon, \\
\sum_{J \subset U ; J \Phi I I}\|\phi(J)\|<\epsilon,
\end{gathered}
$$

We have now

$$
\begin{aligned}
V(U) \geqq & \sum_{J \subset U}\|\phi(J)\|-\epsilon \\
\geqq & \sum_{J \subset W_{n} \cap U}\|\phi(J)\|+\sum_{J \subset G_{n}}\|\phi(J)\|-\epsilon \\
\geqq & \sum_{J \subset W_{n} \cap U}\|\phi(J)\|+\sum_{I \subset G_{n}} \sum_{J \subset I}\|\phi(J)\|-\epsilon \\
= & V\left(W_{n} \cap U\right)+\left[\sum_{J \subset W_{n} \cap U}\|\phi(J)\|-V\left(W_{n} \cap U\right)\right] \\
& +V\left(G_{n}\right)+\sum_{I \subset G_{n}}\left[\sum_{J \subset I}\|\phi(J)\|-\|\phi(I)\|\right] \\
& +\left[\sum_{I \subset G_{n}}\|\phi(I)\|-V\left(G_{n}\right)\right]-\epsilon .
\end{aligned}
$$

By (3.7) and (3.8), we have 


$$
V(U) \geqq V\left(W_{n} \cap U\right)+V\left(G_{n}\right)-4 \epsilon .
$$

We have $W_{n} \cap U \supset U-G$, and hence

$$
V(U) \geqq \mu(U-G)+V\left(G_{n}\right)-4 \epsilon
$$

and, as $n \rightarrow \infty$, also

$$
V(U) \geqq \mu(U-G)+V(U \cap G)-4 \epsilon .
$$

Since $\epsilon>0$ is arbitrary we conclude that

$$
V(U) \geqq \mu(U-G)+V(U \cap G) .
$$

The same holds for $\mu_{r}, \mu_{r}^{+}, \mu_{r}^{-}$. Thereby (3.x) is proved. Note that the inclusions $G_{n} \subset U \cap G, G_{n} \subset \bar{G}_{n} \subset G_{n+1}$, imply $U-G \subset U-\bar{G}_{n}$, where $U-\bar{G}_{n} \in \$$, and

$$
\left(U-\bar{G}_{n}\right) \cap G_{n}=\varnothing, \quad U \supset\left(U-\bar{G}_{n}\right) \cup G_{n}, \quad n=1,2, \cdots
$$

Therefore, by (2.i), we have

$$
V(U) \geqq V\left(G_{n}\right)+V\left(U-\bar{G}_{n}\right) \geqq V\left(G_{n}\right)+\mu(U-G),
$$

and, as $n \rightarrow \infty$, also

$$
V(U) \geqq V(G \cap U)+\mu(U-G) .
$$

This argument gives a new proof of (3.x).

Note that for every set $B \in \mathfrak{B}$ the measure $\mu(B)$ is the infimum of $V(G)$ for $G \in \Re, G \supset B$ and, by definition, $\mu(B)=\operatorname{Inf} V(G)$ for all $G \supset B, G \in \oiint$. By (3.i), there is a sequence $G_{n}, n=1,2, \cdots$, with $G_{n} \in\left(\$, G_{n} \supset G_{n+1}, G_{n} \supset B\right.$, such that $\mu(B)=\lim \mu\left(G_{n}\right)$ as $n \rightarrow \infty$. If $\tilde{G}=\lim G_{n}=\bigcap_{n} G_{n}$, then $B \subset \widetilde{G}$, and $\mu(B)$ $=\mu(\widetilde{G})=\lim \mu\left(G_{n}\right)$ as $n \rightarrow \infty$. Now $\widetilde{G}$ is a $G_{\delta}$-set in the topology of $A$ defined by the collection $(5)$. In the usual terminology (cf. [4, pp. 224-231]), $\mu$ is said to be a regular measure. The same holds for $\mu_{r}, \mu_{r}^{+}, \mu_{r}^{-}$. Thus (3.x) can be reinforced by saying:

(3.xi) Under the same hypotheses of (3.x) the restrictions of $\mu, \mu_{r}, \mu_{r}^{+}, \mu_{r}^{-}$in $B$ are all regular measures.

Finally, if we denote by $\nu$ the vector-valued set function defined on $\mathfrak{M}$ by $\nu=\left(\nu_{1}, \cdots, \nu_{k}\right), \nu_{r}=\mu_{r}^{+}-\mu_{r}^{-}, r=1, \cdots, k$, we may conclude, in the terminology of $[4$, p. 117$]$, with the statement:

(3.xii) Under the same hypotheses as in (3.x) the components $\nu_{r}$ of the restriction of $\nu$ on $\mathfrak{B}$ are signed measures, $r=1, \cdots, k$.

Note that for every set $G \in(\$)$, the vector-valued function $\nu$ has the same values as the function $\mathfrak{B}$ defined in $\$ 1$. Thus, we have extended $\mathfrak{B}$ to the $\sigma$-algebra $\mathfrak{B}$ in such a way that each component $\nu_{r}$ is a signed measure. 
4. Jordan decomposition of the signed measures $\nu_{r}$. It is convenient to take into consideration the "interior part" $I^{0}$ of each of the sets $I$ in the topology defined on $A$ by the collection (S). We shall now require a slightly stronger version of axiom $(\phi)$ concerning quasi additivity, namely

$\left(\phi^{\prime}\right)$ Given $G \in(S)$ nonempty and $\epsilon>0$, there is a number $\eta=\eta(\epsilon, G)$ such that if $D_{0}=[I]$ is any system $D_{0} \in \mathfrak{D}_{G}$ with $\delta\left(D_{0}, G\right)<\eta$, then there is also a number $\lambda=\lambda\left(\epsilon, D_{0}\right)>0$ such that, for every system $D=[J]$ with $\delta(D)<\lambda$ we have

$$
\begin{aligned}
& \left(\phi_{1}^{\prime}\right) \sum_{I \in D_{0}}\left\|\sum_{J \subset I^{0}} \phi(J)-\phi(I)\right\|<\epsilon, \\
& \left(\phi_{2}^{\prime}\right) \sum^{\prime}\|\phi(J)\|<\epsilon, \\
& \text { where } \sum^{\prime} \text { ranges over all } J \in D \text { not completely contained in any } I^{0}, \\
& I \in D_{0} \text {. }
\end{aligned}
$$

An analogous requirement can be made for quasi subadditivity. We do not modify the terminology since, with the new requirements, we are simply treating the collection $\left\{I^{0}\right\}$ as the new collection $\{I\}$. Thus all statements proved for $(\phi)$ hold also for $\left(\phi^{\prime}\right)$. We will use requirement $\left(\phi^{\prime}\right)$ in (4.iii). In particular, Theorem (1.v) holds under the new hypothesis, and we shall refer to it, as usual, in the sequel.

(4.i) Under requirements $\left(\mathrm{a}^{\prime}\right),(\mathrm{b}),(\mathrm{c}),(\mathrm{d}),(\phi),\left(\mathrm{H}^{\prime}\right), V(A)<+\infty$, for each $r=1,2, \cdots, k$, there is a decomposition of $A$ into two measurable disjoint sets $A_{r}^{+}, A_{\bar{r}}, A_{r}^{+} \cup A_{r}^{-}=A, A_{r}^{+} \cap A_{r}^{-}=\varnothing$, such that $A_{r}^{+}$is "positive," and $A_{r}^{-}$is negative," i.e., for every set $F \in \mathfrak{B}$ we have $\nu_{r}\left(A_{r}^{+} \cap F\right) \geqq 0, \nu_{r}\left(A_{r}^{-} \cap F\right) \leqq 0($ Hahn decomposition of $A$ relatively to $\nu_{r}$ ).

This theorem is a consequence of (3.xii) and [4, p. 121].

For every set $B \in \mathfrak{B}$ let us put

$$
\nu_{r}^{+}=\nu_{r}\left(B \cap A_{r}^{+}\right), \quad \nu_{r}^{-}=-\nu_{r}\left(B \cap A_{r}^{-}\right), \quad \nu_{r}^{*}=\nu_{r}^{+}+\nu_{r}^{-} .
$$

Then for every set $B \in \mathfrak{B}$ we have $\nu_{r}^{+} \geqq 0, \nu_{r}^{-} \geqq 0, \nu_{r}^{*} \geqq 0$, and

$$
\nu_{r}(B)=\nu_{r}^{+}(B)-\nu_{r}^{-}(B), \quad \nu_{r}^{*}(B)=\nu_{r}^{+}(B)+\nu_{r}^{-}(B), \quad r=1, \cdots, k .
$$

In the terminology of $\left[4\right.$, p. 122], $\nu_{r}^{+}, \nu_{r}^{-}, \nu_{r}^{*}$ are the upper, lower, and total variations of $\nu_{r}$ respectively. Note that, for every set $B \in \mathscr{B}$, we have

$$
\begin{array}{lrl}
\nu_{r}^{+}\left(B \cap A_{r}^{+}\right)=\nu_{r}\left(B \cap A_{r}^{+}\right) \geqq 0, & \nu_{r}^{+}\left(B \cap A_{r}^{-}\right)=0, & \nu_{r}^{-}\left(B \cap A_{r}^{+}\right)=0, \\
\nu_{r}^{-}\left(B \cap A_{r}^{-}\right)=-\nu_{r}\left(B \cap A_{r}^{-}\right) \geqq 0, & r=1, \cdots, k .
\end{array}
$$

Note that the functions $\nu_{r}^{+}, \nu_{r}^{-}, \nu_{r}^{*}$ are measures in $\mathscr{B}[4$, p. 123]. Note that relations (4.2) are similar to the ones 


$$
\begin{aligned}
& \nu_{r}(B)=\mu_{r}^{+}(B)-\mu_{r}^{-}(B), \\
& \mu_{r}(B)=\mu_{r}^{+}(B)+\mu_{r}^{-}(B),
\end{aligned}
$$

and we will prove (4.iii) that, under condition $\left(\phi^{\prime}\right), \mu_{r}^{+}=\nu_{r}^{+}, \mu_{r}^{-}=\nu_{r}^{-}, \mu_{r}=\nu_{r}^{*}$. Under the sole condition $(\phi)$ no identification is possible between these measures as we will show by examples. The following inequalities hold:

(4.ii) Under the same hypotheses as in (4.i) we have $0 \leqq \nu_{r}^{+} \leqq \mu_{r}^{+}, 0 \leqq \nu_{r}^{-} \leqq \mu_{r}^{-}$, $0 \leqq \nu_{r}^{*} \leqq \mu_{r}^{*}, r=1, \cdots, k$.

Proof. For every set $B \in \mathfrak{B}$, we have

$$
\nu_{r}^{+}(B)=\nu_{r}\left(B \cap A^{+}\right)=\mu_{r}^{+}\left(B \cap A^{+}\right)-\mu_{r}^{-}\left(B \cap A^{+}\right), \quad \nu_{r}^{+}(B)=\nu_{r}^{+}\left(B \cap A^{+}\right) .
$$

Since $\mu_{r}^{-}\left(B \cap A^{+}\right) \geqq 0$, we have $\mu_{r}^{+}\left(B \cap A^{+}\right) \geqq \nu_{r}^{+}\left(B \cap A^{+}\right)$, and finally

$$
\mu_{r}^{+}(B)=\mu_{r}^{+}\left(B \cap A^{+}\right)+\mu_{r}^{+}\left(B \cap A^{-}\right) \geqq \nu_{r}\left(B \cap A^{+}\right)=\nu_{r}^{+}(B) .
$$

Analogously, we can prove that $\mu_{r}^{+}\left(B \cap A^{-}\right) \geqq 0$,

$$
\mu_{r}^{-}\left(B \cap A^{-}\right) \geqq \nu_{r}^{-}\left(B \cap A^{-}\right), \quad \mu_{r}^{-}(B) \geqq \nu_{r}^{-}(B) .
$$

Finally, we have

$$
\mu_{r}(B)-\nu_{r}^{*}(B)=\left[\mu_{r}^{+}(B)-\nu_{r}^{+}(B)\right]+\left[\mu_{r}^{-}(B)-\nu_{r}^{-}(B)\right] \geqq 0 .
$$

We shall now use $\left(\phi^{\prime}\right)$ to prove

(4.iii) Under hypotheses $\left(\mathrm{a}^{\prime}\right),(\mathrm{b}),(\mathrm{c}),(\mathrm{d}),\left(\phi^{\prime}\right),\left(\mathrm{H}^{\prime}\right), V(A)<+\infty$, we have $\mu_{r}^{+}=\nu_{r}^{+}, \mu_{r}^{-}=\nu_{r}^{-}, \mu_{r}=\nu_{r}^{*}$ for every set $B \in \mathfrak{B}$ and $r=1, \cdots, k$. Thus for every set $B \in \mathfrak{B}$ we have $\mu_{r}^{+}=\nu_{r}\left(B \cap A_{r}^{+}\right), \mu_{r}^{-}=-\nu_{r}\left(B \cap A_{r}^{-}\right), \nu_{r}^{*}=\mu_{r}=\mu_{r}^{+}+\mu_{r}^{-}$, and $\nu_{r}$ admits the Jordan decomposition $\nu_{r}=\mu_{r}^{+}-\mu_{r}^{-}$.

Proof. Given $r=1, \cdots, k$, and $\epsilon>0$, let $\mu=\mu(\epsilon, A)$ be the number considered in (1.v), let $D_{0}=[I]$ be any system, $D_{0} \in \mathcal{D}$, with $\delta\left(D_{0}, A\right)<\mu$, and $\lambda$ the corresponding number $\lambda=\lambda\left(\epsilon, D_{0}\right)$ of $\left(\phi^{\prime}\right)$. Then we denote by $G, G^{+}, G^{-}$, the sets which are the unions of all $I^{0}$ with $I \in D_{0}$, or $I \in D_{0}, \phi_{r}(I) \geqq 0$, or $I \in D_{0}$, $\phi_{r}(I)<0$, respectively, and where $I^{0}$ is taken in the topology $(S)$ Then $G, G^{+}$, $G^{-} \in(B), G^{+} \cap G^{-}=\varnothing, G^{+} \cup G^{-}=G$, and

$$
\mid \begin{array}{ll}
\sum \phi_{r}(I)-\mathfrak{B}_{r}(A) \mid<\epsilon, & \left|\sum\right| \phi_{r}(I)\left|-V_{r}(A)\right|<\epsilon, \\
\left|\sum \phi_{r}^{+}(I)-V_{r}^{+}(A)\right|<\epsilon, & \left|\sum \phi_{r}^{-}(I)-V_{r}^{-}(A)\right|<\epsilon,
\end{array}
$$

where $\sum$ ranges over all $I \in D_{0}$, i.e., over all $I^{0} C G$. Nevertheless, in the last two sums we could just suppose that $\sum$ ranges over all $I \in D_{0}$ with $I^{0} \subset G^{+}$, or $I^{0} \subset G^{-}$respectively.

Let $\mu=\mu(\epsilon, G), \mu^{+}=\mu\left(\epsilon, G^{+}\right), \mu^{-}=\mu\left(\epsilon, G^{-}\right)$be the numbers defined in (1.v) and $\nu=\nu(\mu, G), \nu^{+}=\nu\left(\mu^{+}, G^{+}\right), \nu^{-}=\nu\left(\mu^{-}, G^{-}\right)$, the corresponding numbers defined in $\left(\mathrm{d}_{3}\right)$. Let $\nu^{\prime}=\min \left[\lambda, \nu, \nu^{+}, \nu^{-}\right]$, and $D=[J]$ be any system $D \in \mathfrak{D}$ with $\delta(D, A)<\nu^{\prime}$. Then we have 


$$
\begin{array}{ll}
\left|\sum_{J \subset G} \phi_{r}(J)-\mathfrak{B}_{r}(G)\right|<\epsilon, & \left|\sum_{J \subset G}\right| \phi_{r}(J)\left|-V_{r}(G)\right|<\epsilon, \\
\left|\sum_{J \subset G} \phi_{r}^{+}(J)-V_{r}^{+}(G)\right|<\epsilon, & \left|\sum_{J \subset G} \phi_{r}^{-}(J)-V_{r}^{-}(G)\right|<\epsilon, \\
\left|\sum_{J \subset G^{+}} \phi_{r}(J)-\mathfrak{B}_{r}\left(G^{+}\right)\right|<\epsilon, & \left|\sum_{J \subset G^{+}}\right| \phi_{r}(J)\left|-V_{r}\left(G^{+}\right)\right|<\epsilon, \\
\left|\sum_{J \subset G^{+}} \phi_{r}^{+}(J)-V_{r}^{+}\left(G^{+}\right)\right|<\epsilon, & \left|\sum_{J \subset G^{+}} \phi_{r}^{-}(J)-V_{r}^{-}\left(G^{+}\right)\right|<\epsilon, \\
\left|\sum_{J \subset G^{-}} \phi_{r}(J)-\mathfrak{B}_{r}\left(G^{-}\right)\right|<\epsilon, & \left|\sum_{J \subset G^{-}}\right| \phi_{r}(J)\left|-V_{r}(G)\right|<\epsilon, \\
\left|\sum_{J \subset G^{-}} \phi_{r}^{+}(J)-V_{r}^{+}\left(G^{-}\right)\right|<\epsilon, & \left|\sum_{J \subset G^{-}} \phi_{r}^{-}(J)-V_{r}^{-}\left(G^{-}\right)\right|<\epsilon,
\end{array}
$$

and also

$$
\sum_{I \subset G}\left|\sum_{J \in I^{\circ}} \phi_{r}(J)-\phi_{r}(I)\right|<\epsilon, \quad \sum_{I \subset G}\left|\sum_{J \subset I^{0}}\right| \phi_{r}(J)|-| \phi_{r}(I)||<\epsilon,
$$

$$
\sum_{I \subset G}\left|\sum_{J \subset I^{0}} \phi_{r}^{+}(J)-\phi_{r}^{+}(I)\right|<\epsilon, \quad \sum_{I \subset G}\left|\sum_{J \subset I^{0}} \phi_{r}^{-}(J)-\phi_{r}^{-}(I)\right|<\epsilon .
$$

Now, if we consider those $J, I$ with $J \in D, I \in D_{0}, J \subset I^{0}$, with $\phi_{r}(J) \leqq 0$, $\phi_{r}(I) \geqq 0$, or $\phi_{r}(J) \geqq 0, \phi_{r}(I) \leqq 0$, we deduce from the last two inequalities

$$
\begin{aligned}
& \sum_{I \subset \sigma^{+}}\left|\sum_{J \subset I^{0}} \phi_{r}^{-}(J)-0\right|<\epsilon, \\
& \sum_{I \subset G^{-}}\left|\sum_{J \subset I^{0}} \phi_{r}^{+}(J)-0\right|<\epsilon,
\end{aligned}
$$

and hence,

$$
\begin{gathered}
\sum_{J \subset G^{+}} \phi_{r}^{-}(J)<\epsilon, \\
\sum_{J \subset G^{-}} \phi_{r}^{+}(J)<\epsilon .
\end{gathered}
$$

By virtue of the corresponding relations (4.4), we have

$$
V_{r}^{-}\left(G^{+}\right)<2 \epsilon, \quad V_{r}^{+}\left(G^{-}\right)<2 \epsilon .
$$

On the other hand, by virtue of the corresponding relations (4.4), (4.5), (4.6), we have 


$$
\begin{aligned}
0= & V_{r}(A)-V_{r}^{+}(A)-V_{r}^{-}(A) \leqq V_{r}(A)-V_{r}^{+}\left(G^{+}\right)-V_{r}^{-}\left(G^{-}\right) \\
= & {\left[V_{r}(A)-\sum_{I \subset G}\left|\phi_{r}(I)\right|\right]-\sum_{I \subset G}\left[\sum_{J \subset I^{0}}\left|\phi_{r}(J)\right|-\left|\phi_{r}(I)\right|\right] } \\
& +\sum_{J \subset G^{+}} \phi_{r}^{-}(J)+\sum_{J \subset G^{+}}\left[\phi_{r}^{+}(J)-V_{r}^{+}\left(G^{+}\right)\right] \\
& +\sum_{J \subset \sigma^{-}} \phi_{r}^{+}(J)+\sum_{I \subset G^{-}}\left[\phi_{r}^{-}(J)-V_{r}^{-}\left(G^{-}\right)\right] \\
<\epsilon & +\epsilon+\epsilon+\epsilon+\epsilon+\epsilon=6 \epsilon .
\end{aligned}
$$

Thus,

$$
0 \leqq V_{r}(A)-V_{r}^{+}\left(G^{+}\right)-V_{r}^{-}\left(G^{-}\right)<6 \epsilon,
$$

hence

$$
\begin{aligned}
0 & \leqq \mu_{r}^{+}\left(A-G^{+}\right)+\mu_{r}^{-}\left(A-G^{-}\right)=\mu_{r}^{+}(A)+\mu_{r}^{-}(A)-\mu_{r}^{+}\left(G^{+}\right)-\mu^{-}\left(G^{-}\right) \\
& =V_{r}(A)-V_{r}^{+}\left(G^{+}\right)-V_{r}^{-}\left(G^{-}\right)<6 \epsilon,
\end{aligned}
$$

and finally

$$
\mu_{r}^{+}\left(A-G^{+}\right)<6 \epsilon, \quad \mu_{r}^{-}\left(A-G^{-}\right)<6 \epsilon .
$$

Let $B$ be any set $B \in \mathfrak{B}$. Thus, $B \cap G^{+} \in \mathfrak{B}, B \cap G^{-} \in \mathfrak{B}$. There is a sequence $g_{n}, n=1,2, \cdots$, of sets $g_{n} \in G$ such that $g_{n} \supset g_{n+1}, g_{n} \supset B \cap G^{+}, V_{r}^{+}\left(g_{n}\right)$ $\rightarrow \mu_{r}^{+}\left(B \cap G^{+}\right), V_{r}^{-}\left(g_{n}\right) \rightarrow \mu_{r}^{-}\left(B \cap G^{+}\right)$. As a consequence, we have successively $g_{n} \cap G^{+} \in \mathcal{H}, \quad g_{n} \cap G^{+} \supset g_{n+1} \cap G^{+}, \quad g_{n} \cap G^{+} \supset B \cap G^{+}, \quad V_{r}^{+}\left(g_{n} \cap G^{+}\right) \rightarrow \mu_{r}^{+}\left(B \cap G^{+}\right)$, $V_{r}\left(g_{n} \cap G^{+}\right) \rightarrow \mu_{r}^{+}\left(B \cap G^{+}\right), V_{r}^{-}\left(g_{n} \cap G^{+}\right) \rightarrow \mu_{r}^{-}\left(B \cap G^{+}\right)$, and finally

$$
\begin{aligned}
\mu_{r}^{+}\left(B \cap G^{+}\right) & =\lim V_{r}^{+}\left(g_{n} \cap G^{+}\right), \\
\nu_{r}\left(B \cap G^{+}\right) & =\lim \left[V_{r}^{+}\left(g_{n} \cap G^{+}\right)-V_{r}^{-}\left(g_{n} \cap G^{+}\right)\right],
\end{aligned}
$$

and also

$$
\mu_{r}^{+}\left(B \cap G^{+}\right)-\nu_{r}\left(B \cap G^{+}\right)=\lim V_{r}^{-}\left(g_{n} \cap G^{+}\right) .
$$

Thus

$$
\begin{aligned}
0 \leqq & \mu_{r}^{+}\left(B \cap G^{+}\right)-\nu_{r}\left(B \cap G^{+}\right) \leqq V_{r}^{-}\left(G^{+}\right), \\
0 \leqq & \mu_{r}^{+}(B)-\nu_{r}^{+}(B) \\
= & \mu_{r}^{+}\left(B \cap G^{+}\right)+\mu_{r}^{+}\left(B \cap\left(A-G^{+}\right)\right)-\nu_{r}^{+}\left(B \cap G^{+}\right)-\nu_{r}^{+}\left(B \cap\left(A-G^{+}\right)\right) \\
= & {\left[\mu_{r}^{+}\left(B \cap G^{+}\right)-\nu_{r}\left(B \cap G^{+}\right)\right]+\nu_{r}^{-}\left(B \cap G^{+}\right) } \\
& \quad+\left[\mu_{r}^{+}\left(B \cap\left(A-G^{+}\right)\right)-\nu_{r}^{+}\left(B \cap\left(A-G^{+}\right)\right)\right] \\
\leqq & V_{r}^{-}\left(G^{+}\right)+\mu_{r}^{-}\left(B \cap G^{+}\right)+\mu_{r}^{+}\left(B \cap\left(A-G^{+}\right)\right) \leqq 2 V_{r}^{-}\left(G^{+}\right)+\mu_{r}^{+}\left(A-G^{+}\right) \\
< & 4 \epsilon+6 \epsilon=10 \epsilon .
\end{aligned}
$$

Since $\epsilon>0$ is arbitrary, we conclude that $\mu_{r}^{+}(B)=\nu_{r}^{+}(B)$ for every $B \in \mathfrak{B}$. 
Analogously, we can prove that $\mu_{r}^{-}(B)=\nu_{r}^{-}(B)$. Finally, we have

$$
\mu_{r}(B)-\nu_{r}^{*}(B)=\left[\mu_{r}^{+}(B)-\nu_{r}^{+}(B)\right]+\left[\mu_{r}^{-}(B)-\nu_{r}^{-}(B)\right]=0 .
$$

The last part of statement (4.iii) is now a trivial consequence of Theorem (4.i) and relations (4.2). Thereby (4.iii) is completely proved.

REMARK. If we replace hypothesis $\left(\phi^{\prime}\right)$ by the slightly weaker hypothesis $(\phi)$, then (4.iii) does not hold necessarily, i.e., the hypotheses $\left(a^{\prime}\right),(b),(c)$, (d), $(\phi),\left(\mathrm{H}^{\prime}\right), V(A)<+\infty$ do not imply the conclusion of (4.iii). This can be seen by the following example, which even satisfies (e) and (g).

Let $A=[-1 \leqq x \leqq 1]$ with the usual topology, and $(S=\mathfrak{U}$ be the collection of all open sets $G$ open in $A$. Let $\{I\}=\left\{I_{r n}\right\}$ be the collection of all closed intervals $I_{r n}=\left[r 2^{-n},(r+1) 2^{-n}\right], \quad r=-2^{n}, \quad-2^{n}+1, \cdots, 2^{n}-1$, $\mathfrak{D}=\left\{D_{n}, n=0,1,2, \cdots\right\}$ the collection of all finite families $D_{n}$ $=\left[I_{r n}, r=-2^{n},-2^{n}+1, \cdots, 2^{n}-1\right]$. Thus for every set $G$ open in $A, D_{n G}$ is the subfamily of all (closed) $I_{r n}$ with $I_{r n} \subset G, r=-2^{n},-2^{n}+1, \cdots, 2^{n}-1$, and $\mathfrak{D}_{G}=\left[D_{n G}, n=1,2, \cdots\right]$. Finally, we take $\delta\left(D_{n G}, G\right)=2^{-n}$, and $k=1$, $\phi\left(I_{r n}\right)=0$ if $r \neq-1,0, \phi\left(I_{r n}\right)=-1$ if $r=-1, \phi\left(I_{r n}\right)=+1$ if $r=0$. Then, if $0 \in G$, we have $V(G)=2, \mathfrak{B}(G)=0, V^{+}(G)=V^{-}(G)=1$; if $0 \notin G$, we have $V=\mathfrak{B}=V^{+}=V^{-}=0$. Thus, $\mathfrak{B}(G)=0, V^{+}(G)=V^{-}(G)$ for all $G$ and, by (3.1) $\mu^{+}(M)=\mu^{-}(M)$ for all subsets $M$ of $A$, hence, by (3.2) and (4.1), $\nu(M)$ $=\nu^{+}(M)=\nu^{-}(M)=0$ for all subsets $M$ of $A$, while $\mu^{+}(M)=\mu^{-}(M)=1$ for every $M$ with $0 \in M$.

Given any set $B \in \mathfrak{B}$, we shall denote by $[H]$ any finite decomposition $[H]=\left[H_{1}, \cdots, H_{n}\right], \quad B=H_{1} \cup H_{2} \cup \cdots \cup H_{n}$ of $B$ into disjoint sets $H_{1}, \cdots, H_{n} \in \mathfrak{B}$.

(4.iv) Under the same hypotheses as (4.iii) and for every set $B \in \mathfrak{B}$, we have

$$
\mu(B)=\operatorname{Sup}_{[H]} \sum_{H \in[H]}\left[\sum_{r=1}^{k} \mu_{r}^{2}(H)\right]^{1 / 2}=\operatorname{Sup}_{[H]} \sum_{H \in[H]}\left[\sum_{r=1}^{k} \nu_{r}^{2}(H)\right]^{1 / 2} .
$$

Proof. From (3.iii), (3.x) and the definitions, we have

$$
\sum_{H \in[H]}\left[\sum_{r=1}^{k} \nu_{r}^{2}(H)\right]^{1 / 2} \leqq \sum_{H \in[H]}\left[\sum_{r=1}^{k} \mu_{r}^{2}(H)\right]^{1 / 2} \leqq \sum_{H \in[H]} \mu(H)=\mu(B) .
$$

Given $\epsilon>0$ let $G \in B$ be chosen in such a way that $B \subset G, \mu(B) \leqq V(G)<\mu(B)$ $+\epsilon$, and analogous relations hold for $\mu_{r}, r=1, \cdots, k$. Let $\mu=\mu(\epsilon, G)>0$ be the number defined as in (1.v), let $D_{0}=[I]$ be a system of $N$ sets $I$ with $D_{0} \in \mathscr{D}_{G}, \delta\left(D_{0}, G\right)<\mu$, and let $\lambda=\lambda\left(\epsilon, D_{0}, G\right)$ be the number defined as in (1.v). Then we certainly have

$$
\left|V(G)-\sum_{I \in D_{0}}\|\phi(I)\|\right|<\epsilon, \quad\left|V_{r}(G)-\sum_{I \in D_{0}}\right| \phi_{r}(I)||<\epsilon, r=1, \cdots, k .
$$


Let $G^{\prime}=U I^{0}$, where $U$ ranges over all $I \in D_{0}$. Then $G^{\prime} \subset G, G^{\prime} \in(s)$. Let $\mu^{\prime}=\mu\left(\epsilon, G^{\prime}\right)>0, \mu^{\prime}(I)=\mu\left(\epsilon / N, I^{0}\right), I \in D_{0}$, be numbers defined as in (1.v), let $\nu^{\prime}=\nu\left(\mu^{\prime}, G^{\prime}\right)>0, \nu^{\prime}(I)=\nu\left(\mu^{\prime}(I), I^{0}\right)>0, \nu^{\prime \prime}=\nu(\lambda, G)>0$ be numbers defined as in $\left(\mathrm{d}_{8}\right)$, and $D=[J]$ be any system with $D \in \mathfrak{D}=\mathfrak{D}_{\Delta}, \delta(D, A)$ $<\min \left[\nu^{\prime}, \nu^{\prime}(I), I \in D_{0}, \nu^{\prime \prime}\right]$. Let $D_{\sigma^{\prime}}, D_{I^{0}}, D_{G}$ be the unions of all $J \in D$ which are respectively in $G^{\prime}, I^{0}, G,\left(I \in D_{0}\right)$. Then we have

$$
\delta\left(D_{G^{\prime}}, G^{\prime}\right)<\mu^{\prime}, \quad \delta\left(D_{I^{0}}, I^{0}\right)<\mu^{\prime}(I), \quad \delta\left(D_{G}, G\right)<\lambda,
$$

and thus

$$
\begin{gathered}
\left|V\left(G^{\prime}\right)-\sum_{J \subset G^{\prime}}\|\phi(J)\|\right|<\epsilon, \quad\left|V_{r}\left(G^{\prime}\right)-\sum_{J \subset G^{\prime}}\right| \phi_{r}(J)||<\epsilon, \\
\left|V\left(I^{0}\right)-\sum_{J \subset I^{0}}\|\phi(J)\|\right|<\epsilon / N, \quad\left|V_{r}\left(I^{0}\right)-\sum_{J \subset I^{0}}\right| \phi_{r}(J)||<\epsilon / N, \\
\sum_{I \in D_{0}}\left|\sum_{J \subset I}\|\phi(J)\|-\|\phi(I)\|\right|<\epsilon, \quad \sum_{I \in D_{0}}\left|\sum_{J \subset I^{0}}\right| \phi_{r}(J)|-| \phi_{r}(I)||<\epsilon .
\end{gathered}
$$

We have

$$
\mu(B)<V(G)+\epsilon<\sum_{I \in D_{0}}\|\phi(I)\|+2 \epsilon=\sum_{I \in D_{0}}\left[\sum_{r=1}^{k} \phi_{r}^{2}(I)\right]^{1 / 2}+2 \epsilon .
$$

If we denote by $\xi_{r}(I)$ the difference under $\mid$ in the sixth relation (4.10), we have also, by substitution and Minkowski's inequality,

$$
\begin{aligned}
\mu(B) & <\sum_{I \in D_{0}}\left[\sum_{r=1}^{k}\left\{\sum_{J \subset I^{0}}\left|\phi_{r}(J)\right|-\xi_{r}(I)\right\}^{2}\right]^{1 / 2}+2 \epsilon \\
& \leqq \sum_{I \in D_{0}}\left[\sum_{r=1}^{k}\left\{\sum_{J \subset I^{0}}\left|\phi_{r}(J)\right|+\left|\xi_{r}(I)\right|\right\}^{2}\right]^{1 / 2}+2 \epsilon \\
& \leqq \sum_{I \in D_{0}}\left[\sum_{r=1}^{k}\left\{\sum_{J \subset I^{0}}\left|\phi_{r}(J)\right|\right\}^{2}\right]^{1 / 2}+\sum_{I \in D_{0}}\left[\sum_{r=1}^{n} \xi_{r}^{2}(I)\right]^{1 / 2}+2 \epsilon \\
& \leqq \sum_{I \in D_{0}}\left[\sum_{r=1}^{k}\left\{\sum_{J \subset I^{0}}\left|\phi_{r}(J)\right|\right\}^{2}\right]^{1 / 2}+\sum_{r=1}^{k} \sum_{I \in D_{0}}\left|\xi_{r}(I)\right|+2 \epsilon \\
& \leqq \sum_{I \in D_{0}}\left[\sum_{r=1}^{k}\left\{\sum_{J \subset I^{0}}\left|\phi_{r}(J)\right|\right\}^{2}\right]^{1 / 2}+(k+2) \epsilon .
\end{aligned}
$$

If we denote by $\zeta_{r}(I)$ the difference under || in the fourth relation (4.10), we have also, by the same argument, 


$$
\begin{aligned}
\mu(B) & <\sum_{I \in D_{0}}\left[\sum_{r=1}^{k}\left\{V_{r}\left(I^{0}\right)-\zeta_{r}(I)\right\}^{2}\right]^{1 / 2}+(k+2) \epsilon \\
& <\sum_{I \in D_{0}}\left[\sum_{r=1}^{k} V_{r}^{2}\left(I^{0}\right)\right]^{1 / 2}+(2 k+2) \epsilon .
\end{aligned}
$$

We have $I^{0}, G^{\prime} \in\left(S\right.$ and hence, by (3.iv), $\mu_{r}\left(I^{0}\right)=V_{r}\left(I^{0}\right)$, and finally

$$
\mu(B)<\sum_{I \in D_{0}}\left[\sum_{r=1}^{k} \mu_{r}^{2}\left(I^{0}\right)\right]^{1 / 2}+(2 k+2) \epsilon .
$$

For every $I \in D_{0}$ let $I^{\prime}, I^{\prime \prime}$ be the sets $I^{\prime}=I^{\circ} \cap B, I^{\prime \prime}=I^{0}-B$, and let $K=G-G^{\prime}, \quad M=K \cap B=B-G^{\prime}=B-\cup I^{0}$. We have $I^{\prime}, I^{\prime \prime}, K, M \in \mathfrak{B}$, $I^{0}=I^{\prime} \cup I^{\prime \prime}, \mu_{r}\left(I^{0}\right)=\mu_{r}\left(I^{\prime}\right)+\mu_{r}\left(I^{\prime \prime}\right)$, and

$$
\begin{aligned}
\mu\left(U I^{\prime \prime}\right)=\sum \mu\left(I^{\prime \prime}\right) \leqq \mu(G-B) & =\mu(G)-\mu(B)<\epsilon, \\
\mu_{r}\left(U I^{\prime \prime}\right)=\sum \mu_{r}\left(I^{\prime \prime}\right) \leqq \mu_{r}(G-B)=\mu_{r}(G)-\mu_{r}(B)<\epsilon, & \quad r=1, \cdots, k,
\end{aligned}
$$

where $U$ and $\sum$ range over all $I \in D_{0}$. We have, by (4.11)

$$
\mu(B)<\sum_{I \in D_{0}}\left[\sum_{r=1}^{k}\left\{\mu_{r}\left(I^{\prime}\right)+\mu_{r}\left(I^{\prime \prime}\right)\right\}^{2}\right]^{1 / 2}+(2 k+2) \epsilon,
$$

and, by repeating the same reasoning above,

$$
\mu(B)<\sum_{I \in D_{0}}\left[\sum_{r=1}^{k} \mu_{r}^{2}\left(I^{\prime}\right)\right]^{1 / 2}+(3 k+2) \epsilon .
$$

Finally we have

$$
\mu(B)<\sum_{I \in D_{0}}\left[\sum_{r=1}^{k} \mu_{r}^{2}\left(I^{\prime}\right)\right]^{1 / 2}+\left[\sum_{r=1}^{k} \mu_{r}^{2}(K)\right]^{1 / 2}+(3 k+2) \epsilon,
$$

where the $N$ sets $I^{\prime}$ and $K$ form a decomposition of $B$ into $N+1$ disjoint sets of $B$. This proves that

$$
\mu(B) \leqq \operatorname{Sup}_{[H]} \sum_{H \in[H]}\left[\sum_{r=1}^{k} \mu_{r}^{2}(H)\right]^{1 / 2} .
$$

This result together with (4.8) proves the first part of (4.iv).

To prove the second part of (4.iv) let us observe that (4.12) actually states that there exists a decomposition $[H]$ of $B$ with

$$
\mu(B)<\sum_{H \in[H]}\left[\sum_{r=1}^{k} \mu_{r}^{2}(H)\right]^{1 / 2}+(3 k+2) \epsilon .
$$

Let $H_{r}^{+}=H A_{r}^{+}, H_{r}^{-}=H A_{r}^{-}, r=1, \cdots, k$, and 


$$
H_{i_{1} i_{2} \cdots i_{k}}=H_{1}^{ \pm} \cap H_{2}^{ \pm} \cap \cdots \cap H_{k}^{ \pm}, \quad i_{1}, i_{2}, \cdots, i_{k}=1,2,
$$

where we take $H_{1}^{+}$if $i_{1}=1, H_{1}^{-}$if $i_{1}=2$, and analogously for $H_{2}^{ \pm}, \cdots, H_{\mathbf{k}}^{ \pm}$. Thus

$$
H=\underset{i_{1}, \cdots, i_{k}=1,2}{\bigcup} H_{i_{1} \cdots i_{k}}
$$

where $U$ ranges over the $2^{k}$ disjoint sets $H_{i_{1}} \ldots i_{k}$ all in $\mathfrak{B}$. If $\sum^{\prime}$ denotes any sum ranging over all these sets, we have

$$
\begin{aligned}
\mu(B) & <\sum_{H \in[H]}\left[\sum_{r=1}^{k}\left\{\sum^{\prime} \mu_{r}\left(H_{i_{1}} \ldots i_{k}\right)\right\}^{2}\right]^{1 / 2}+(3 k+2) \epsilon \\
& \leqq \sum_{H \in[H]} \sum^{\prime}\left[\sum_{r=1}^{k} \mu_{r}^{2}\left(H_{i_{1}} \ldots i_{k}\right)\right]^{1 / 2}+(3 k+2) \epsilon
\end{aligned}
$$

where now $\mu_{r}\left(H_{i_{1}} \cdots i_{k}\right)= \pm \nu_{r}\left(H_{i_{1}} \ldots i_{k}\right)$. Hence

$$
\mu(B) \leqq \sum_{H \in[H]} \sum^{\prime}\left[\sum_{r=1}^{k} \nu_{r}^{2}\left(H_{i_{1}} \ldots i_{k}\right)\right]^{1 / 2}+(3 k+2) \epsilon,
$$

and, by the same argument above, we prove the second part of (4.iv).

5. Radon-Nikodym derivatives. The measures $\mu_{r}^{+}, \mu_{r}^{-}$and the signed measures $\nu_{r}$ are absolutely continuous with respect to $\mu_{r}$, and $\mu_{r}, \mu_{r}^{+}, \mu_{r}^{-}, \nu_{r}$ are absolutely continuous with respect to $\mu, r=1,2, \cdots, k$. Indeed, by (3.1), (3.2), (3.3), we have $\left|\nu_{r}\right|=\left|\mu_{r}^{+}-\mu_{r}^{-}\right| \leqq \mu_{r}^{+}+\mu_{r}^{-}=\mu_{r} \leqq \mu$. Hence the RadonNikodym derivatives

$$
\begin{array}{rrrrl}
\theta_{r}(w)=\frac{d \nu_{r}}{d \mu}, & \beta_{r}(w)=\frac{d \mu_{r}}{d \mu}, & \beta_{r}^{+}(w)=\frac{d \mu_{r}^{+}}{d \mu}, & \beta_{r}^{-}(w)=\frac{d \mu_{r}^{-}}{d \mu} . \\
\gamma_{r}(w)=\frac{d \nu_{r}}{d \mu_{r}}, & \gamma_{r}^{+}(w)=\frac{d \mu_{r}^{+}}{d \mu_{r}}, & \gamma_{r}^{-}(w)=\frac{d \mu_{r}^{-}}{d \mu_{r}}, & r=1, \cdots, k,
\end{array}
$$

exist $(\mu)$-a.e. and $\left(\mu_{r}\right)$-a.e. in $A$ respectively, are measurable functions in the measure spaces $(A, \mathfrak{B}, \mu),\left(A, \mathfrak{B}, \mu_{r}\right)$ respectively, and we have $-1 \leqq \theta_{r}, \gamma_{r} \leqq 1$, $0 \leqq \beta_{r}, \beta_{r}^{+}, \beta_{r}^{-}, \gamma_{r}^{+}, \gamma_{r}^{-} \leqq 1$. We shall also denote by $\theta(w)$ the vector valued function $\theta(w)=\left(\theta_{1}, \cdots, \theta_{k}\right)$. have

(5.i) Under the hypotheses $\left(\mathrm{a}^{\prime}\right),(\mathrm{b}),(\mathrm{c}),(\mathrm{d}),\left(\phi^{\prime}\right),\left(\mathrm{H}^{\prime}\right), V(A)<+\infty$, we (a)

$$
\begin{array}{ccc}
\beta_{r}=\beta_{r}^{+}+\beta_{r}^{-}, & \theta_{r}=\beta_{r}^{+}-\beta_{r}^{-}, \quad \beta_{r}^{+}=\gamma_{r}^{+} \beta_{r}, \quad \beta_{r}^{-}=\gamma_{r}^{-} \beta_{r}, & (\mu) \text {-a.e. in } A ; \\
\gamma_{r}^{+}+\gamma_{r}^{-}=1, & \gamma_{r}=\gamma_{r}^{+}-\gamma_{r}^{-}, &
\end{array}
$$




$$
\begin{array}{lll}
\beta_{r}^{+} \beta_{r}^{-}=0, & \left|\theta_{r}\right|=\left|\beta_{r}^{+}-\beta_{r}^{-}\right|=\left|\beta_{r}^{+}+\beta_{r}^{-}\right|=\beta_{r}, & (\mu) \text {-a.e. in } A ; \\
\gamma_{r}^{+} \gamma_{r}^{-}=0, & \left|\gamma_{r}\right|=\left|\gamma_{r}^{+}-\gamma_{r}^{-}\right|=\gamma_{r}^{+}+\gamma_{r}^{-}=1, & \left(\mu_{r}\right) \text {-a.e. in } A ;
\end{array}
$$

and, either

$$
\gamma_{r}^{+}=1, \quad \gamma_{r}^{-}=0, \quad \text { or } \gamma_{r}^{+}=0, \quad \gamma_{r}^{-}=1, \quad\left(\mu_{r}\right) \text {-a.e. in } A .
$$

Proof. The relations $\beta_{r}=\beta_{r}^{+}+\beta_{r}^{-}, \theta_{r}=\beta_{r}^{+}-\beta_{r}^{-},(\mu)$-a.e. follow from $\mu_{r}$ $=\mu_{r}^{+}+\mu_{r}^{-}$(3.ii) and $\nu_{r}=\mu_{r}^{+}-\mu_{r}^{-}$(definition (3.2)) respectively. The relations $\beta_{r}^{+}=\gamma_{r}^{+} \beta_{r}, \beta_{r}^{-}=\gamma_{r}^{-} \beta_{r},(\mu)$-a.e. follow from the chain rule on Radon-Nikodym derivatives.

The equalities $\gamma_{r}^{+}+\gamma_{r}^{-}=1$ and $\gamma_{r}=\gamma_{r}^{+}-\gamma_{r}^{-},\left(\mu_{r}\right)$-a.e., follow from $\mu_{r}^{+}+\mu_{r}^{-}$ $=\mu_{r}$ and $\nu_{r}=\mu_{r}^{+}-\mu_{r}^{-}$again. Thus (a) is proved.

We will derive the equality $\beta_{r}^{+} \beta_{r}^{-}=0$, ( $\mu$-a.e., from (4.i) and (4.iii). Indeed, we have $\beta_{r}^{-}=0$, $(\mu)$-a.e. on $A_{r}^{+}$, and hence $\theta_{r}=\beta_{r}^{+}-\beta_{r}^{-}=\beta_{r}^{+}=\beta_{r}^{+}+\beta_{r}^{-}=\beta_{r}$, ( $\mu)$-a.e. on $A_{r}^{+}$; we have $\beta_{r}^{+}=0$, $(\mu)$-a.e. on $A_{r}^{-}$, and hence $-\theta_{r}=\beta_{r}^{-}-\beta_{r}^{+}=\beta_{r}^{-}$ $=\beta_{r}^{+}+\beta_{r}^{-}=\beta_{r},(\mu)-$ a.e., on $A_{r}^{-}$. Thus $\left|\theta_{r}\right|=\left|\beta_{r}^{+}-\beta_{r}^{-}\right|=\beta_{r}^{+}+\beta_{r}^{-}=\beta_{r}$, $(\mu)$-a.e. as in $A$.

$\mid$ The relations $\gamma_{r}^{+} \gamma_{r}^{-}=0,\left|\gamma_{r}\right|=\left|\gamma_{r}^{+}-\gamma_{r}^{-}\right|=\gamma_{r}^{+}+\gamma_{r}^{-}=1,\left(\mu_{r}\right)$-a.e. are proved similarly. Thus $\gamma_{r}^{+}=1, \gamma_{r}^{-}=0$, or $\gamma_{r}^{+}=0, \gamma_{r}^{-}=1$, $\left(\mu_{r}\right)$-a.e., and (5.i) is proved.

(5.ii) Under the same hypotheses as in (5.i) we have

$$
\theta_{1}^{2}+\cdots+\theta_{k}^{2}=\|\theta\|^{2}=\beta_{1}^{2}+\cdots+\beta_{k}^{2}=1,
$$

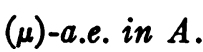

This statement is a consequence of $[6, \mathrm{p} .318]$ and (5.i).

Let $D=[I]$ be any system $D \in \mathfrak{D}$ and, for every $I \in \mathfrak{D}$, let us consider the set $I^{0} \subset I, I^{0} \in(B)$, where $I^{0}$ is taken in the topology $(S)$. Let $G=U I^{0}, G \in \mathbb{S}$, where $U$ ranges over all $I \in D$. Let $\eta(w), w \in A$, be the vector function

$$
\eta(w)=\nu\left(I^{0}\right) / \mu\left(I^{0}\right) \text { if } w \in I^{0}, I \in D ; \quad \eta(w)=0 \text { if } w \in A-G .
$$

Then $\eta(w)=\left(\eta_{1}, \cdots, \eta_{k}\right)$ and $\|\eta(w)\| \leqq 1$ for all $w \in A$. Also, $\eta(w)$ is constant in each of the disjoint sets $I^{0}$ with $I \in D$, and $A-G$, all belonging to $\mathfrak{B}$, and hence $\eta(w)$ is $\mu$-measurable in $A$.

(5.iii) Under the same hypotheses as in (5.i) we have

$$
\lim _{\delta(D) \rightarrow 0}(A) \int\|\theta(w)-\eta(w)\|^{2} d \mu=0 .
$$

Proof. Given $\epsilon>0$, let $\mu=\mu(\epsilon, A)>0$ be defined as in (1.v), and $D=[I]$ any system $D \in \mathcal{D}$ of $N$ sets $I \in\{I\}$ with $\delta(D, A)<\mu$. Let $\lambda=\lambda(\epsilon, D)$ be defined as in (1.v). Let $\mu(I)=\mu\left(\epsilon / N, I^{0}\right)$ be defined as in (1.v) for each $I \in D$, and $\nu(I)=\nu\left(\mu(I), I^{0}\right)$ as in $\left(\mathrm{d}_{3}\right)$. Finally let $\nu^{\prime}=\min [\mu, \lambda, \nu(I)]$ for all $I \in D$. Let $D^{\prime}=[J] \in \mathcal{D}$ be any system with $\delta\left(D^{\prime}, A\right)<\nu^{\prime}$. Then we have 


$$
\begin{array}{r}
\sum_{I \in D}\left\|\sum_{J \in I^{0}} \phi(J)-\phi(I)\right\|<\epsilon, \quad\left|V(A)-\sum_{I \in D}\|\phi(I)\|\right|<\epsilon, \\
\left\|V\left(I^{0}\right)-\sum_{J \in I^{0}} \phi(J)\right\|<\epsilon / N,\left|V\left(I^{0}\right)-\sum_{J \in I^{0}}\|\phi(J)\|\right|<\epsilon / N
\end{array}
$$

for all $I \in D$. By $I^{0} \in(S)$, and (3.iv) we have

$$
\begin{aligned}
\sum_{I \in D}\left\|\nu\left(I^{0}\right)-\phi(I)\right\| & =\sum_{I \in D}\left\|\mathfrak{B}\left(I^{0}\right)-\phi(I)\right\| \\
& \leqq \sum_{I \in D}\left\|\mathfrak{B}\left(I^{0}\right)-\sum_{J \subset I^{0}} \phi(J)\right\|+\sum_{I \in D}\left\|\sum_{J \subset I^{0}} \phi(J)-\phi(I)\right\| \\
& \leqq N(\epsilon / N)+\epsilon=2 \epsilon .
\end{aligned}
$$

If $\sum$ denotes any sum ranging over all $I \in D$, we have

$$
\text { (A) } \begin{aligned}
\int\|\theta-\eta\|^{2} d \mu & =(A) \int\|\theta\|^{2} d \mu-2 \sum_{r=1}^{k}(A) \int \theta_{r} \eta_{r} d \mu+(A) \int\|\eta\|^{2} d \mu \\
& =\mu(A)-2 \sum \sum_{r=1}^{k}\left(I^{0}\right) \int \theta_{r} \eta_{r} d \mu+\sum\left(I^{0}\right) \int\|\eta\|^{2} d \mu \\
& =\mu(A)-2 \sum\left\|\nu\left(I^{0}\right)\right\|^{2} / \mu\left(I^{0}\right)+\sum\left\|\nu\left(I^{0}\right)\right\|^{2} / \mu\left(I^{0}\right) \\
& =\mu(A)-\sum\left\|\nu\left(I^{0}\right)\right\|^{2} / \mu\left(I^{0}\right) \\
& =\mu(A)-\sum\left\|\nu\left(I^{0}\right)\right\|+\sum\left[\left\|\nu\left(I^{0}\right)\right\|-\left\|\nu\left(I^{0}\right)\right\|^{2} / \mu\left(I^{0}\right)\right] \\
& =\mu(A)-\sum\left\|\nu\left(I^{0}\right)\right\|+\sum\left[\left\|\nu\left(I^{0}\right)\right\| / \mu\left(I^{0}\right)\right]\left[\mu\left(I^{0}\right)-\nu\left(I^{0}\right)\right] \\
& \leqq 2\left[\mu(A)-\sum\left\|_{\nu}\left(I^{0}\right)\right\|\right] \\
& \leqq 2\left[V(A)-\sum\|\phi(I)\|\right]+2 \sum\|\| \phi(I)\|-\| \nu\left(I^{0}\right) \| \mid .
\end{aligned}
$$

Thus, by (5.3) and (5.4) we have

$$
\text { (A) } \int\|\theta-\eta\|^{2} d \mu<2 \epsilon+2 \cdot 2 \epsilon=6 \epsilon
$$

for every $D \in \mathcal{D}$ and $\delta(D, A)<\mu$. Thereby, (5.iii) is proved. Statement (5.iii) extends $[2$, p. 361] and $[3$, p. 150].

6. The integrals $\Im$ and $\Im_{0}$. In [1] we have considered a set $A$, a collection (5) made up of the only set $A$, a collection $\{I\}$ of sets $I \subset A$, a collection $\mathfrak{D}$ of finite systems $D=[I]$ of sets $I \in\{I\}$ satisfying (b), a mesh $\delta(D)=\delta(D, A)$ satisfying $\left(\mathrm{d}_{1}\right)$ and $\left(\mathrm{d}_{2}\right)$, a vector function $\phi(I)=\left(\phi_{1}, \cdots, \phi_{k}\right), I \in\{I\}$, satisfying $(\phi)$ with respect to $\delta(D)$ and $\mathfrak{D}$ (i.e., quasi additive). If $V=V(\|\phi\|)$ $=V(A)<+\infty$, then all functions $\phi, \phi_{r},\|\phi\|,\left|\phi_{r}\right|, \phi_{r}^{+}, \phi_{r}^{-}$are quasi additive.

Let $T: p=p(w), w \in A, p=\left(x_{1}, \cdots, x_{m}\right)$, be any mapping from $A$ into a given set $K \subset E_{m}$. Then for every $I \in\{I\}$ let $\omega(I)=$ Osc $(T, I)$, and, for every 
$D=[I] \in \mathcal{D}$, let $\omega(D)=\max \omega(I)$ for all $I \in D$. We have supposed in [1] that $\delta(D)$ has been chosen in such a way that

$$
\omega(D) \leqq \delta(D)
$$

for all $D \in \mathfrak{D}$.

This is actually a continuity requirement for $T$ in $A$.

Let $p=\left(x_{1}, \cdots, x_{m}\right)$ denote any point $p \in E_{m}$ as above, $q=\left(q_{1}, \cdots, q_{k}\right)$ any point $q \in E_{k}$, and $\mathfrak{S}$ the unit sphere in $E_{k}$, or $\mathfrak{S}=\left[q \in E_{k},\|q\|=1\right]$. Let $f(p, q), p \in K \subset E_{m}, q \in E_{k}$, be any function of $(p, q)$ defined for all $(p, q)$ $\in K \times E_{k}$, such that

$\left(\mathrm{f}_{1}\right) f$ is a bounded and uniformly continuous function of $(p, q)$ in $K \times \mathbb{S}$;

$\left(\mathrm{f}_{2}\right) f(p, t q)=t f(p, q)$ for all $t \geqq 0, p \in K, q \in E_{k}$.

For every $I \in\{I\}$ we may choose arbitrarily a point $\tau \in I$ and consider the set function

$$
\Phi(I)=f[p(\tau), \phi(I)], \quad I \in\{I\} .
$$

We have proved in [1]:

(6.i) Under hypotheses $\mathbb{B}=A,(\mathrm{~b}),\left(\mathrm{d}_{1}\right),\left(\mathrm{d}_{2}\right),(\phi),(\omega),(\mathrm{f})$ and $V(A)<+\infty$, the scalar set function $\Phi(I), I \in\{I\}$, satisfies $(\phi)$ (i.e., $\Phi$ is quasi additive, and given $\epsilon>0$, the numbers $\eta(\epsilon), \lambda\left(\epsilon, D_{0}\right)$ of $(\phi)$ can be determined independently of the choice of $\tau$ in each set $I, I \in D, D \in D$.

(6.ii) Under the same hypotheses as in (6.i), the following limit exists and is finite,

$$
\Im=\Im(f, T, \phi)=\lim _{\delta(D) \rightarrow 0} \sum_{I \in D} f[p(\tau), \phi(I)],
$$

where $D=[I] \in \mathcal{D}, \tau$ is any point $\tau \in I$, and $\Im$ is independent of the choice of $\tau$ on each $I \in D$.

$\Im$ is said to be the $\Im$-integral of $f$ on the mapping $T$ with respect to the quasi additive function $\phi$.

In the present paper, by using hypothesis (a), we could replace $A$ in (6.i) and (6.ii), by any set $G$ of the collection $\mathbb{B}$, and then the integral above could be thought of as a set function $I(G, f, T, \phi), G \in(B)$.

Also, by using hypotheses $\left(a^{\prime}\right),\left(\phi^{\prime}\right)$ (instead of $(a)$ and $\left.(\phi)\right)$ we may define $I$ by means of another limit, analogous to the one in (6.1), as it was done in [3]. Indeed, the following theorem holds:

(6.iii) Under hypotheses $\left(\mathrm{a}^{\prime}\right),(\mathrm{b}),(\mathrm{c}),(\mathrm{d}),\left(\phi^{\prime}\right),\left(\mathrm{H}^{\prime}\right), V(A)<+\infty,(\omega),(\mathrm{f})$, we have

$$
\Im(f, T, \phi)=\lim _{\delta(D) \rightarrow 0} \sum_{I \in D} f\left[p(\tau), \nu\left(I^{0}\right)\right] .
$$

Proof. Let $M>0$ be a number such that $\mu(A)=V(A) \leqq M-1,|f(p, q)| \leqq$ 
$M-1$ for all $p \in K, q \in \mathfrak{S}$. Given $0<\epsilon \leqq 1$, let $\epsilon_{1}=\epsilon / 15 M$, and $\rho, 0<\rho<\epsilon_{1} / 14$, a number such that $\left|f(p, q)-f\left(p, q^{\prime}\right)\right|<\epsilon_{1}$, for all $p \in K, 1-\epsilon_{1} \leqq\|q\|,\left\|q^{\prime}\right\| \leqq 1$, $\left\|q-q^{\prime}\right\| \leqq 14 \rho$. Let $\sigma=\rho^{6} / 48 k$, let $\mu=\mu(\sigma, A), 0 \leqq \mu \leqq \sigma$, be the number defined in (1.v), and $D=[I] \in \mathcal{D}$ any system with $\delta(D, A)<\mu$. Let $\lambda=\lambda(\sigma, D)$, $0<\lambda \leqq \mu$, be a number defined as in (1.v), and let $D^{\prime}=[J]$ be any system with $\delta\left(D^{\prime}, A\right)<\lambda$.

For any $I \in \mathfrak{D}$ and $J \in D$ let $\alpha(I), \beta(J)$ be the unit vectors $\alpha(I)$ $=\phi(I) /\|\phi(I)\|, \beta(J)=\phi(J) /\|\phi(J)\|$. Let $\sum_{I}, \sum_{J}, \sum^{(I)}, \sum^{\prime}$ denote as usual sums ranging over all $I \in D$, all $J \in D^{\prime}$, all $J \in D^{\prime}$ with $J \subset I^{0}$, all $J \in D^{\prime}$ with $J \nsubseteq I^{0}$ for any $I \in D$, respectively, and let $\sum^{*}$ be any sum ranging over all $J \in D^{\prime}$ with $J \subset I^{0}$ for some $I \in D$ and $\|\beta(J)-\alpha(I)\| \geqq \rho^{2}$. Then, by Lemma (5.i) of the previous paper [1] (where $\epsilon$ is now replaced by $\rho^{2}$ ), we have

$$
\begin{aligned}
& \sum^{*}\|\phi(J)\|<\rho^{2}, \quad \sum^{\prime}\|\phi(J)\|<\rho^{2}, \\
& \sum_{I} \sum(I)\|\phi(J)\|\|\beta(J)-\alpha(I)\|^{2}<\rho^{2} \text {, } \\
& \sum_{I} \mid \sum(I)\|\phi(J)\|-\|\phi(I)\|<\rho^{2}, \quad \sum_{I}\left\|\sum(I) \phi(J)-\phi(I)\right\|<\rho^{2} \text {, } \\
& \left\|\mathfrak{B}(A)-\sum_{I} \phi(I)\right\|<\rho^{2}, \quad\left|V(A)-\sum_{I}\|\phi(I)\|\right|<\rho^{2}, \\
& \left\|\mathfrak{B}(A)-\sum_{J} \phi(J)\right\|<\rho^{2}, \quad\left|V(A)-\sum_{J}\|\phi(J)\|\right|<\rho^{2} .
\end{aligned}
$$

Since $D^{\prime}=[J] \in \mathscr{D}$ can be taken with mesh $\delta\left(D^{\prime}, A\right)$ arbitrarily small, we deduce from the fourth and fifth relations (6.1) that

$$
\sum_{I}\left\|\mathfrak{B}\left(I^{0}\right)-\phi(I)\right\| \leqq \rho^{2}, \quad \sum_{I}\left|V\left(I^{0}\right)-\|\phi(I)\|\right| \leqq \rho^{2},
$$

For every $I$ let us denote by $\sum^{*(I)}, \sum^{0(I)}$ sums ranging over all $J \in D^{\prime}$, $J \subset I^{0}$, with $\|\beta(J)-\alpha(I)\| \geqq \rho$, or $\|\beta(J)-\phi(I)\|<\rho$ respectively. Let us denote by $D_{2}^{\prime}, D_{2}^{\prime \prime}, D_{2}^{\prime \prime \prime}, D_{2}^{\text {iv }}$ the subcollections of all $I \in D$ such that, respectively

$$
\begin{aligned}
\left\|\sum^{(I)} \phi(J)-\mathfrak{B}\left(I^{0}\right)\right\| & \geqq \rho\|\phi(I)\|, \\
\left|\sum^{(I)}\|\phi(J)\|-V\left(I^{0}\right)\right| & \geqq \rho\|\phi(I)\|, \\
\left|\|\phi(I)\|-V\left(I^{0}\right)\right| & \geqq \rho\|\phi(I)\|, \\
\sum^{*(I)}\|\phi(J)\| & \geqq \rho\|\phi(I)\| .
\end{aligned}
$$

Let $D_{2}=D_{2}^{\prime} \cup D_{2}^{\prime \prime} \cup D_{2}^{\prime \prime \prime} \cup D_{2}^{i v}$ and $D_{1}=D-D_{2}$. For every $I \in D_{1}$ we have

$$
\begin{aligned}
& \mathfrak{B}\left(I^{0}\right)=\sum^{(I)} \phi(J)+\rho_{1}\|\phi(I)\| \quad \text { with }\left\|\rho_{1}\right\|<\rho, \\
& V\left(I^{0}\right)=\sum(I)\|\phi(J)\|+\rho_{2}\|\phi(I)\| \text { with }\left|\rho_{2}\right|<\rho \text {, } \\
& V\left(I^{0}\right)=\|\phi(I)\|+\rho_{3}\|\phi(I)\| \quad \text { with }\left|\rho_{3}\right|<\rho, \\
& \sum *(I)\|\phi(J)\|=\rho_{4}\|\phi(I)\| \\
& \text { with }\left|\rho_{4}\right|<\rho \text {, }
\end{aligned}
$$

and, for every $J \in D^{\prime}, J \subset I^{0}, I \in D_{1}$, we have

with

$$
\phi(J)=\alpha\|\phi(J)\|+\psi(J)
$$




$$
\begin{aligned}
\sum^{(I)}\|\psi(J)\| & =\left(\sum^{0(I)}+\sum^{*(I)}\right)\|\phi(J)-\alpha\| \phi(J)\|\| \\
& \leqq \sum^{0(I)} \rho\|\phi(J)\|+2 \sum^{*(I)}\|\phi(J)\| \\
& \leqq \rho \sum^{(I)}\|\phi(J)\|+2 \sum^{*(I)}\|\phi(J)\| \\
& \leqq \rho\left[V\left(I^{0}\right)-\rho_{2}\|\phi(I)\|\right]+2 \rho_{4}\|\phi(I)\| \\
& \leqq \rho\|\phi(I)\|\left(1-\rho_{2}+\rho_{3}\right)+2 \rho_{4}\|\phi(I)\| \\
& =\|\phi(I)\|\left[\rho\left(1-\rho_{2}+\rho_{3}\right)+2 \rho_{4}\right]<5 \rho\|\phi(I)\|,
\end{aligned}
$$

and hence

$$
\sum^{(I)} \psi(J)=5 \rho_{5}\|\phi(I)\| \quad \text { with } \quad 0 \leqq\left\|\rho_{5}\right\|<\rho .
$$

We have also, by (6.3) and (6.2), (6.1),

$$
\begin{aligned}
\sum_{I \in D_{2}}\|\phi(I)\| \leqq & \rho^{-1} \sum_{I \in D}\left[\left\|\sum^{I} \phi(J)-\mathfrak{B}\left(I^{0}\right)\right\|\right. \\
& +\left|\sum^{(I)}\|\phi(J)\|-V\left(I^{0}\right)\right|+\left|\|\phi(I)\|-V\left(I^{0}\right)\right|+\sum^{*(I)}\|\phi(J)\| \mid \\
& \leqq \rho^{-1}\left[2 \rho^{2}+2 \rho^{2}+\rho^{2}+\rho^{2}\right]=6 \rho .
\end{aligned}
$$

Finally, by combining (6.4) and (6.5), we have, for every $I \in D_{1}$,

$$
\begin{aligned}
& \nu\left(I^{0}\right)=\mathfrak{B}\left(I^{0}\right)=\sum^{(I)} \phi(J)+\rho_{1}\|\phi(I)\| \\
&=\alpha \sum^{(I)}\|\phi(J)\|+\sum^{(I)} \psi(J)+\rho_{1}\|\phi(I)\| \\
&=\alpha\left[V\left(I^{0}\right)-\rho_{2}\|\phi(I)\|\right]+5 \rho_{5}\|\phi(I)\|+\rho_{1}\|\phi(I)\| \\
&=\|\phi(I)\|\left[\alpha\left(1+\rho_{3}-\rho_{2}\right)+\rho_{1}+5 \rho_{5}\right], \\
& \mu\left(I^{0}\right)=V\left(I^{0}\right)=\|\phi(I)\|\left(1+\rho_{3}\right), \\
& \gamma(I)=\nu\left(I^{0}\right) / \mu(I)=\left[\alpha\left(1+\rho_{3}+\rho_{2}\right)+\rho_{1}+5 \rho_{5}\right]\left(1+\rho_{3}\right)^{-1}, \\
&\|\gamma(I)-\alpha(I)\|=\left\|\left(-\alpha \rho_{2}+\rho_{1}+5 \rho_{5}\right)\left(1+\rho_{3}\right)^{-1}\right\|<14 \rho^{2} .
\end{aligned}
$$

Since $\|\alpha\|=1,14 \rho \leqq \epsilon_{1}$, we have $\|\gamma\| \geqq 1-\epsilon_{1}$, and also $\|\gamma\| \leqq 1$ since $\mu$ is the total variation of $\nu$. Thus for all $I \in D_{1}$ we have

$$
|f[p(\tau), \alpha(I)]-f[p(\tau), \gamma(I)]|<\epsilon_{1} .
$$

We have now, since $V\left(I^{0}\right)=\mu\left(I^{0}\right), \mathfrak{B}\left(I^{0}\right)=\nu\left(I^{0}\right)$, and by force of $\left(\mathrm{f}_{2}\right)$,

$$
\begin{aligned}
|\Delta|=\mid & \left|\sum_{I} f[p(\tau), \phi(I)]-\sum_{I} f\left[p(\tau), \nu\left(I^{0}\right)\right]\right| \\
= & \left|\sum_{I} f[p(\tau), \alpha(I)]\|\phi(I)\|-\sum_{I} f[p(\tau), \gamma(I)] \mu\left(I^{0}\right)\right| \\
\leqq & \sum_{I \in D_{1}}|f[p(\tau), \alpha(I)]-f[p(\tau), \gamma(I)]|\|\phi(I)\| \\
& +\sum_{I \in D_{1}}|f[p(\tau), \gamma(I)]|\|\phi(I)\|-\mu\left(I^{0}\right) \mid \\
& +\sum_{I \in D_{2}}\left|f[p(\tau), \alpha(I)]\|\phi(I)\|+\sum_{I \in D_{2}}\right| f[p(\tau), \gamma(I)] \mu\left(I^{0}\right) .
\end{aligned}
$$


By the definition of $\rho$ we have

$$
\begin{aligned}
|\Delta| & \leqq \epsilon_{1} \sum_{I}\|\phi(I)\|+M \rho+M \cdot 6 \rho+M\left(6 \rho+\rho^{2}\right) \\
& \leqq \epsilon_{1}\left[V+\rho^{2}\right]+14 M \rho<15 M \epsilon_{1}=\epsilon
\end{aligned}
$$

for all $D \in \mathfrak{D}$ with $\delta(D, A)<\mu$. Thus $\Delta \rightarrow 0$ as $\delta(D) \rightarrow 0$ and, thereby, (6.iii) is proved.

(6.iv) Under hypotheses (a'), (b), (c), (d), ( $\left.\phi^{\prime}\right),\left(\mathrm{H}^{\prime}\right), V(A)<\infty,(\omega)$, (f), the function $f[p(w), \theta(w)], w \in A$, is $\mu$-integrable in $A$.

Proof. Since $p(w) \in K,\|\theta(w)\|=1$, ( $\mu)$-a.e. in $A$, and $f$ is bounded in $K \times \mathfrak{S}$, we conclude that $f[p(w), \theta(w)]$ is defined and bounded $(\mu)$-a.e. in $A$ and, hence, it is sufficient to prove that the same function is $\mu$-measurable in $A$.

If $D_{n}, n=1,2, \cdots$, is any sequence of systems $D_{n} \in \mathfrak{D}$ with $\delta\left(D_{n}, A\right) \rightarrow 0$ as $n \rightarrow \infty$, and we denote by $\eta_{n}(w), w \in A$, the corresponding sequence of functions defined in $\S 5$, i.e., $\eta_{n}(w)=\left[\eta_{n 1}, \cdots, \eta_{n k}\right], \eta_{n}(w)=\nu\left(I^{0}\right) / \mu\left(I^{0}\right)$ for every $w \in I^{0}, I \in D_{n}, \eta_{n}(w)=0$ otherwise, we have $(A) \int\left\|\theta(w)-\eta_{n}(w)\right\|^{2} d \mu \rightarrow 0$ as $n \rightarrow \infty$. Hence, we have also $(A) \int\left\|\theta(w)-\eta_{n}(w)\right\| d \mu \rightarrow 0$ as $n \rightarrow \infty$, and finally $\eta_{n}(w) \rightarrow \theta(w)$ in $(\mu)$-measure in $A$. Finally, there is a subsequence $n_{s}$ of integers $n_{\mathrm{s}} \rightarrow \infty$ such that $\eta_{n},(w) \rightarrow \theta(w)$ as $s \rightarrow \infty(\mu)$-a.e. in $A$. Thus, we may select a ( $\mu$ )-measurable subset $A^{*}$ of $A$ and a sequence $D_{n}, n=1,2, \cdots$, such that $D_{n} \in \mathfrak{D}, \delta\left(D_{n}, A\right)<1 / n, n=1,2, \cdots, \mu\left(A-A^{*}\right)=0,\|\theta(w)\|=1$ for all $w \in A^{*}$, and $\eta_{n}(w) \rightarrow \theta(w)$ as $n \rightarrow \infty$ for all $w \in A^{*}$.

For every $I \in D_{n}$ let us take a point $\tau_{n} \in I^{0}$ and let $p_{n}(w)$, $w \in A$, be the mapping defined by $p_{n}(w)=p\left(\tau_{n}\right)$ for all $w \in I^{0}, I \in D_{n}$, and $p_{n}(w)=p_{0}$ for all $w \in A-G^{\prime}, G^{\prime}=A-\cup I^{0}, \cup$ ranging over all $I \in D_{n}$, where $p_{0}$ is an arbitrary fixed point of $K$. Thus $p_{n}: A \rightarrow K, \eta_{n}: A \rightarrow E_{k}$, and both $p_{n}(w)$ and $\eta_{n}(w)$, $w \in A$, are $(\mu)$-measurable, since they are constant on each of the sets $I^{0} \in \mathscr{B}$, $A-G^{\prime} \in \mathfrak{B}$. Since $f$ is continuous on $K \times E_{k}$ we conclude that $f\left[p_{n}(w), \eta_{n}(w)\right]$, $w \in A$, is $\mu$-measurable in $A, n=1,2, \cdots$.

Let $w_{0}$ be any point $w_{0} \in A^{*}$. Since $\left\|\dot{\theta}\left(w_{0}\right)\right\|=1, \eta_{n}\left(w_{0}\right) \rightarrow \theta\left(w_{0}\right)$, there is an integer $n_{0}=n_{0}\left(w_{0}\right)$ such that $\left\|\eta_{n}\left(w_{0}\right)-\theta\left(w_{0}\right)\right\|<1 / 2$, and hence $1 / 2 \leqq\left\|\eta_{n}\left(w_{0}\right)\right\|$ $\leqq 3 / 2$ and $\eta_{n}\left(w_{0}\right) \neq 0$, for all $n \geqq n_{0}$. As a consequence, $w_{0} \in I^{0}$ for some $I \in D_{n}$, and $\eta_{n}\left(w_{0}\right)=\nu\left(I^{0}\right) / \mu\left(I^{0}\right), p_{n}\left(w_{0}\right)=p\left(\tau_{n}\right), \tau_{n} \in I^{0}$, and $\left\|p_{n}\left(w_{0}\right)-p\left(w_{0}\right)\right\|$ $\leqq \operatorname{Osc}\left[p(w), I^{0}\right] \leqq \omega\left(D_{n}\right) \leqq \delta\left(D_{n}, A\right)<1 / n$ for all $n \geqq n_{0}$. By $(\mathrm{f})$, the function $f$ is uniformly continuous on the set $K \times \Im^{\prime}, \Im^{\prime}=\left[q \mid q \in E_{k}, 1 / 2 \leqq\|q\| \leqq 3 / 2\right]$, and, by $\left[p_{n}\left(w_{0}\right), \eta_{n}\left(w_{0}\right)\right] \in K \times \mathfrak{S}^{\prime},\left[p_{n}\left(w_{0}\right), \eta_{n}\left(w_{0}\right)\right] \rightarrow\left[p\left(w_{0}\right), \theta\left(w_{0}\right)\right] \in K \times \widetilde{S}$, we conclude that $f\left[p_{n}\left(w_{0}\right), \eta_{n}\left(w_{0}\right)\right] \rightarrow f\left[p\left(w_{0}\right), \theta\left(w_{0}\right)\right]$ as $n \rightarrow \infty$, for all $w_{0} \in A^{*}$. Thus $f[p(w), \theta(w)]$ is $\mu$-measurable in $A^{*}$, and, since $\mu\left(A-A^{*}\right)=0$, this function is $\mu$-measurable in $A$. Thereby, (6.iv) is proved.

Under the hypotheses of (6.iv) the function $f[p(w), \theta(w)], w \in A$, is defined $(\mu)$-a.e. in $A$ and is $(\mu)$-integrable in $A$. Hence, the integral

$$
\Im_{0}=\Im_{0}(f, T, \nu)=(A) \int f[p(w), \theta(w)] d \mu
$$


exists and is finite. $\Im_{0}$ is said to be the $\Im$-integral of the function $f$ on the mapping $T$ with respect to the vector valued measure $\nu$. We shall prove in (6.v) that $\Im=\Im_{0}$ under the same hypotheses as above, i.e., the integral $\Im$ defined in (6.ii) has the representation

$$
\Im(f, T, \phi)=(A) \int f[p(w), \theta(w)] d \mu .
$$

REMARK. Let us assume that $(A, B, \nu)$ is any given measure space, where $\nu=\left(\nu_{1}, \cdots, \nu_{k}\right)$ is a vector valued measure with total variation $\mu$ and $\mu(A)$ $<\infty$. If $\theta_{r}(w)=d \nu_{r} / d \mu$ denote the Radon-Nikodym derivative of $\nu_{r}$ with respect to $\mu$, and $\theta(w)=\left[\theta_{1}, \cdots, \theta_{k}\right]$, then $\theta(w)$ is defined $(\mu)$-a.e. in $A$ and $\|\theta(w)\|=1$, $(\mu)$-a.e. in $A$. Also, suppose that $f(p, q), p \in K \subset E_{n}, q \in E_{k}$, is any function satisfying axiom (f), and $p(w), w \in A, p(w) \in K$, a mapping satisfying solely the hypothesis: $f[p(w), \theta(w)], w \in A$, is $\mu$-integrable in $A$. Under these assumptions an integral $\Im_{0}(f, T, \nu)$ exists and is finite. In (6.iv) we have just proved that these assumptions are verified for the measure function $\nu$ defined in $\$ 3$.

(6.v) Under hypotheses (a'), (b), (c), (d), $\left(\phi^{\prime}\right),\left(\mathrm{H}^{\prime}\right), V(A)<\infty,(\omega)$, (f) we have $\Im=\Im_{0}$, i.e. (6.6) holds.

Proof. Let $M>0$ be chosen as in (6.iii) and, given $\epsilon>0$, let $\epsilon_{1}$ and $\rho$ be chosen as in (6.iii). By (6.ii), (6.iii), (5.iii), there is $\lambda, 0<\lambda \leqq \rho$, such that for any finite system $D=[I] \in \mathcal{D}$ with $\delta(D, A)<\lambda$ we have

$$
\begin{array}{r}
\left|\Im(f, T, \phi)-\sum_{I} f\left[p(\tau), \nu\left(I^{0}\right)\right]\right|<\epsilon_{1}, \\
(A) \int\|\theta(w)-\eta(w)\|^{2} d \mu<\rho^{2} \epsilon_{1} .
\end{array}
$$

Let $G=U I^{0}$, where $U$ ranges over all $I \in D$, let $\gamma(I)=\nu\left(I^{0}\right) / \mu\left(I^{0}\right)$ and $\eta(w)$, $w \in A$, be the function defined, as in (5.2), by $\eta(w)=\gamma(I)$ for $w \in I^{0}$, and $\eta(w)=0$ in $A-G$. Also, let $\tilde{p}(w), w \in A$, be the function defined by $\tilde{p}(w)=p(\tau)$ for $w \in I^{0}, \tilde{p}(w)=p(w)$ in $A-G$. Finally, let us observe that, by $(\omega)$, we have $\operatorname{Osc}(T, I)=\omega(I) \leqq \omega(D) \leqq \delta(D, A)<\lambda \leqq \rho$ for every $I \in D$. Let $B \subset A$ be the set of all $w \in A$ with $\|\theta(w)-\eta(w)\|<\rho$ and let $C=A-B$. Then we have $\|\theta-\eta\| \geqq \rho$ in $C$,

$$
\rho^{2} \mu(C) \leqq(A) \int\|\theta-\eta\|^{2} d \mu \leqq \rho^{2} \epsilon_{1}
$$

and finally $\mu(C)<\epsilon_{1}$. Thus we have

$$
\begin{aligned}
& |f[\tilde{p}(w), \eta(w)]-f[\tilde{p}(w), \theta(w)]|<\epsilon_{1}, \\
& |f[\tilde{p}(w), \theta(w)]-f[p(w), \theta(w)]|<\epsilon_{1},
\end{aligned}
$$

for every $w \in B$. Also we have 


$$
\sum_{I} f\left[p(\tau), \nu\left(I^{0}\right)\right]=\sum_{I} f[p(\tau), \gamma(I)] \mu\left(I^{0}\right)=(A) \int f[\tilde{p}(w), \eta(w)] d \mu .
$$

Finally, we have successively

$$
\begin{aligned}
|\Delta|= & \left|\Im(f, T, \phi)-(A) \int f[p(w), \theta(w)] d \mu\right| \\
\leqq & \left|\Im(f, T, \phi)-\sum_{I} f\left[p(\tau), \nu\left(I^{0}\right)\right]\right| \\
& +\left|\sum_{I} f\left[p(\tau), \nu\left(I^{0}\right)\right]-(A) \int f[\tilde{p}(w), \eta(w)] d \mu\right| \\
& +\left|(B) \int\{f[\tilde{p}(w), \eta(w)]-f[\tilde{p}(w), \theta(w)]\} d \mu\right| \\
& +\left|(B) \int\{f[\tilde{p}(w), \theta(w)]-f[p(w), \theta(w)]\} d \mu\right| \\
& +\left|(C) \int f[\tilde{p}(w), \eta(w)] d \mu\right|+\left|(C) \int f[p(w), \theta(w)] d \mu\right| \\
\leqq & \epsilon_{1}+0+\epsilon_{1} \mu(B)+\epsilon_{1} \mu(B)+2 M \mu(C) \\
< & \epsilon_{1}+M \epsilon_{1}+M \epsilon_{1}+2 M \epsilon_{1} \leqq 5 M \epsilon_{1}<\epsilon .
\end{aligned}
$$

Thus, $\Delta \rightarrow 0$ as $\delta(D, A) \rightarrow 0$ and, thereby, (6.v) is proved.

List of axioms: (a), p. 117 ; (a'), p. $124 ;(\mathrm{b})=\left(\mathrm{b}_{1}, \mathrm{~b}_{2}\right)$, p. $115 ;(\mathrm{c})$, p. 117 ; $(\mathrm{d})=\left(\mathrm{d}_{1}, \mathrm{~d}_{2}, \mathrm{~d}_{3}\right)$, p. $115 ;(\mathrm{e})$, p. $120 ;(\mathrm{g})$, p. $123 ;(\phi)=\left(\phi_{1}, \phi_{2}\right)$, p. $115 ;(\psi)$, p. $116 ;\left(\phi^{\prime}\right)$, p. $130 ;(\mathrm{H})=\left(\mathrm{H}_{1}, \mathrm{H}_{2}, \mathrm{H}_{3}\right)$, p. $119 ;\left(\mathrm{H}^{\prime}\right)=\left(\mathrm{H}_{1}, \mathrm{H}_{2}, \mathrm{H}_{3}, \mathrm{H}_{4}\right)$, p. 127 ; $(f)=\left(f_{1}, f_{2}\right)$, p. $140 ;(\omega)$, p. $140 ;(p)$, p. 127 .

Content. Introduction, p. $114 ; \$ 1$, Quasi additive set functions, p. 115; $\S 2$, Connection with a topology in A, p. $117 ; \S 3$, A measure $\mu$ associated to $\phi$, p. $124 ; \S 4$, Jordan decomposition of the signed measures $\nu_{r}$, p. $130 ; \S 5$, RadonNikodym derivatives, p. $137 ; \S 6$, The integrals $\Im$ and $\Im_{0}$, p. 139.

\section{REFERENCES}

1. L. Cesari, Quasi additive set functions and the concept of integral over a variety, Trans. Amer. Math. Soc. vol. 102 (1962), pp. 94-113.

2. - Surface Area, Princeton University Press, 1956.

3. L. Cesari and L. H. Turner, Surface integral and Radon-Nikodym derivatives, Rend. Circ. Mat. Palermo vol. 7 (1958) pp. 143-154.

4. P. R. Halmos, Measure theory, New York, Van Nostrand, 1950.

5. A. Rosenthal, Set functions, Albuquerque, University of New Mexico Press, 1948.

6. H. Whitney, Geometric integration theory, Princeton University Press, 1957.

7. E. Cech, Sur la dimension des espaces parfaitement normaux, Bull. Intern. Acad. Bohème vol. 33 (1932) pp. 38-55.

8. P. Alexandroff and P. Urysohn, Memoire sur les ensembles compacts, Verh. Acad. Amsterdam vol. 14 (1928-1929), pp. 93. 
9. N. Vedenissoff, Sur les fonctions continues dans les espaces topologiques, Fund. Math. vol. 27 (1936) pp. 234-238.

10. T. Nishiura, Analytic theory of continuous transformations, Thesis, Purdue University, 1959.

11. L. H. Turner, Measures induced on a r-algebra by a surface, Duke Math. J. vol. 26 (1959) pp. 501-510.

UNIVERSITY OF MICHIGAN, AnN Arbor, Michigan 